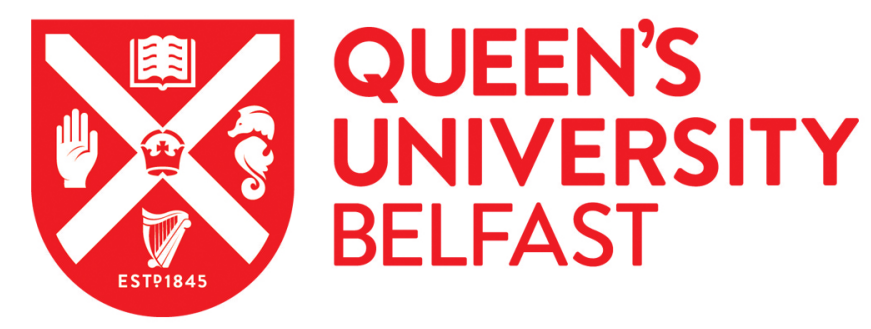

\title{
Multispecies plasma expansion into vacuum: The role of secondary ions and suprathermal electrons
}

Elkamash, I. S., \& Kourakis, I. (2016). Multispecies plasma expansion into vacuum: The role of secondary ions and suprathermal electrons. Physical Review E, 94(5), [053202]. https://doi.org/10.1103/PhysRevE.94.053202

\author{
Published in: \\ Physical Review E
}

Document Version:

Peer reviewed version

Queen's University Belfast - Research Portal:

Link to publication record in Queen's University Belfast Research Portal

Publisher rights

(c) 2016 American Physical Society

\section{General rights}

Copyright for the publications made accessible via the Queen's University Belfast Research Portal is retained by the author(s) and / or other copyright owners and it is a condition of accessing these publications that users recognise and abide by the legal requirements associated with these rights.

Take down policy

The Research Portal is Queen's institutional repository that provides access to Queen's research output. Every effort has been made to ensure that content in the Research Portal does not infringe any person's rights, or applicable UK laws. If you discover content in the Research Portal that you believe breaches copyright or violates any law, please contact openaccess@qub.ac.uk. 
Author copy.

Original published in: Physical Review E. 94 (5), 15 p., 053202 (2016);

DOI : $10.1103 /$ PhysRevE.94.053202.

\title{
Multi-species plasma expansion into vacuum: the role of secondary ions and suprathermal electrons
}

\author{
I. S. Elkamash ${ }^{1,2^{*}}$ and I. Kourakis ${ }^{1}$ \\ 1 Centre for Plasma Physics, Queen's University Belfast, BT7 1 NN Northern Ireland, UK \\ 2 Physics Department, Faculty of Science, Mansoura University, 35516 Mansoura, Egypt
}

(Dated: October 31, 2016)

\begin{abstract}
The self similar expansion of multi-species ion plasma is investigated by a two-ion fluid model with adiabatic equation of state for each ionic species. Our aim is to elucidate the effect of secondary ions on a plasma expansion front, in combination with energetic (suprathermal) electrons in the background, modeled by a kappa-type distribution function. The plasma density, velocity and electric field profile is investigated. It is shown that energetic electrons have a significant effect on the expansion front dynamics, essentially energizing the front, thus enhancing the ion acceleration mechanism. Different special cases are considered, as regards the relative magnitude of the ion mass and/or charge state.
\end{abstract}

\section{INTRODUCTION}

Plasma expansion into vacuum has received constantly growing interest in recent years, in particular due to its relevance with experiments on ultraintense laser pulse interaction with solid targets [1-5], and with applications of high energy ion beams, e.g. for ion acceleration $[6-$ 9], laser-assisted fast ignition scenaria for fusion [10-12]. Experimental low-temperature plasmas [13] and plasmas for medical applications [14-18] (e.g. cancer therapy) involving plasma expansion mechanisms have received increasing attention in the last few years. Various plasma expansion schemes have been modelled via particle-in-cell (PIC) simulations, to validate theoretical models [19-21].

From a theoretical modeling point of view, the main building blocks have been set in the early works of Gurevich et al [22] and later of Allen and coworkers [23]. These were succeeded by a series of remarkable contributions by Mora and coworkers [24-29], including a study of the effect of charge separation, i.e. violation of the charge neutrality hypothesis (plasma approximation), numerically $[25,30]$, for electron-ion plasmas. Interestingly, a bi-Maxwellian (two electron temperature) approach was considered in a number of works [31-33], described the effect of the co-existence of two thermal electron populations. Other analytical studies have focused on kinetic-theoretical considerations for the electron distribution [34], the effect of instabilities [35] and even magnetic field generation [36], among other effects.

Not against physical intuition, and certainly in favor of analytical tractability, the electron background is often tacitly assumed to be isothermal throughout the ionfluid expansion procedure. However, in realistic situations, laser-target interaction certainly generate energetic (suprathermal) electrons, which are energized due to various acceleration mechanisms. These electrons may be characterized by particle distribution functions featuring

\footnotetext{
*E-mail address: elkamashi@gmail.com
}

a long tail in the velocity spectrum, which may significantly deviate from the Maxwellian distribution. One widely accepted paradigm of a long-tailed distribution is the so-called kappa distribution [37, 38], which is known to model a number of situations in Space [39, 40], but also in the laboratory [41]. The kappa approach was adopted in a plasma expansion context only recently, considering superthermal electron effects in electron-ion plasmas [42]. The kappa distribution is characterized by a real parameter $(\kappa)$, whose "smallness" measures deviation from thermal equilibrium: the Maxwell-Boltzmann state is obtained in the infinite $\kappa$ limit. It has been shown that kappa distributions provide excellent fits in a variety of environments, not only in Space [43-47], but also in numerical experiments [48]. The kappa distribution is by now established as an efficient tool for providing correct predictions of observed modifications of, e.g., the plasma sound speed and the associated charge screening length [47], both of which are modified in the presence of energetic electrons [38].

In Space plasmas, it appears that $\kappa$ values between 3 and 6 are ubiquitous in the solar wind [39], and also in planetary magnetospheres [44, 48]. The ability for accurate measurements of particle distributions by sophisticated diagnostic devices onboard spacecraft missions [39] provides a tool for precise characterisation of energetic particle distributions, which seem to establish the fact that the long-tailed distribution observed are well fit by kappa-type theories [40, 46].

In high-power laser-plasma interaction experiments, on the other hand, qualitative evidence based on proton diagnostics [49] suggests that the electron population is not thermalized, while various types of non-Maxwellian situations, e.g. "bump-on-tail" distributions may develop, depending on the surrounding plasma environment. For any target thickness, it is evident that the electrons are in a nonthermal state, for long times (large multiples of the plasma period) [50]. Admittedly, in proton imaging based experiments, it appears that current diagnostic techniques do not allow for an accurate characterization of the electron distribution, hence the electron distribution may often have to be inferred indirectly 
[50]. Suprathermal particles also appear in other types of laser-plasma interaction experiments e.g. in hohlraums [51], and also in fusion plasmas [52]. Therefore, although a link to kappa-type theories may not be rigorously established, in the laboratory, these seem to capture the generic characteristics of electrostatic excitations at least, as observations seem to be compatible with waveforms predicted by related studies [41].

As opposed to the "textbook" two-component (electron-ion) plasma picture, experimentally produced laser plasmas contain several ion species, due to increasingly complicated composite material targets, leading to multicomponent plasma configurations [53-56]. Interestingly, secondary ions (rather than electrons) have recently been argued to contribute to ultrafast collisional plasma heating by electrostatic shocks [57]. The role of a minority secondary ion population on plasma expansion was investigated earlier in a number of studies [31, 5861]. Our work at hand aims at generalizing those earlier models by considering a nonthermal (non-Maxwellian) plasma environment and a finite (arbitrary) admixture of ion components. From first principles, the presence of secondary ions in an expanding plasma is manifested in the appearance of spectral peaks, which are of interest both in experimental diagnostics and for application purposes $[31,59]$. As a simplifying hypothesis, one may assume that the plasma doesn't change its shape during the expansion process and that there is no charge separation. Therefore, the expanding plasma can be studied as a self-similar process. Physically speaking, this assumption is justified if the system's scale is much larger than the characteristic Debye length, and the flow of both ions and electrons is sufficient smooth (laminar) on the ionic timescale. As a matter of fact, the shape of the expanding plasma front has been investigated via numerical simulation in a number of studies in the past [25, 34, 62, 63], which corroborated the general features of the self-similar solution. As a matter of fact, the aforementioned studies have addressed the expansion of a collisionless cold-ion fluid against an electron cloud obeying either a step-like or a Maxwell-Boltzmann distribution. A critical comparison between the analytical (self-similar) solution and the numerical results has confirmed that the self-similar solution can predict the general features of the expansion front but rather fails to predict the actual position of the ion front and of the associated electric field at early times. However, those numerical studies have confirmed that, with the passage of time, plasma motion approaches the self-similar picture quite precisely, regardless of the background electron profile. It is worth mentioning that a series of recent numerical and experimental studies have established a link with particle acceleration mechanisms via spontaneous shock creation during plasma expansion [19-21]. Concluding, the self-similar solution cannot explain the complete process of plasma expansion into vacuum, but it succeeds in capturing the essential features of the problem and bears, in fact, the significant advantage of analytical tractability.
Our aim in this work, is to elucidate the effect of secondary ions on the plasma expansion front, in combination with the role of energetic electrons in the background. A kappa-type electron distribution function $[37,38]$ is adopted. Adopting a self-similar analysis, the evolution of the plasma density, velocity and electric field profile is investigated in detail. It is shown that energetic electrons have a significant effect on the expansion front dynamics, essentially energizing the front, thus enhancing the ion acceleration mechanism. Different special cases are considered, in terms of the relative magnitude of the ion mass and/or charge state.

The layout of this article goes as follows. In Section II, an analytical model is developed and its physical implications are discussed. The self similar expansion mechanism is investigated, adopting different assumptions for the plasma slab, in Section III. The single-ion limiting case (cold- or warm-ion model) is presented, for reference, and the theory is extended to a two-ion fluid model, in the presence of heavier (minority) ions. Finally, Section IV summarizes our results and conclusions.

\section{THE MODEL}

We consider a planar plasma slab consisting of electrons (absolute charge $e$, mass $m_{e}$ ) and two different (positive) ion populations. The two ion species are characterized by their respective mass $m_{j}$, charge $q_{j}=z_{j} e$ and temperature $T_{j}$, as well as their equilibrium density $n_{j, 0}$, respectively (for $j=1,2$ ). The plasma is assumed to be quasineutral, hence, the electron number density inside the slab equals $n_{e, 0}=\sum z_{j, 0} n_{j, 0}$ at equilibrium.

At $t=0$, the plasma is assumed to occupy the negative semi-axis (for $x<0$ ), while vacuum is assumed to occupy the positive semi-axis (for $x>0$ ); here $x$ is the distance measured from the plasma slab, as shown in Fig. 1.

The dynamics of the ions (at $t>0$ ) can be described by the multi-fluid model

$$
\begin{aligned}
\frac{\partial n_{j}}{\partial t}+\frac{\partial}{\partial x}\left(n_{j} u_{j}\right) & =0 \\
m_{j} n_{j}\left(\frac{\partial u_{j}}{\partial t}+u_{j} \frac{\partial u_{j}}{\partial x}\right) & =z_{j} e n_{j} E-\frac{\partial P_{j}}{\partial x}, \\
\frac{\partial E}{\partial x}=4 \pi e\left(z_{1} n_{1}+z_{2} n_{2}-n_{e}\right) &
\end{aligned}
$$

where indices $j=1,2$ denote ion fluid(s) 1 and 2 , respectively. The notation is self-explanatory: $n_{j}$ is the ion-density, $u_{j}$ is the ion velocity, $P_{j}$ is the partial ion pressure, $m_{j}$ is the ion mass, and $z_{j}$ is the ion charge state (for $j=1,2$ ).

The electrons are described by the "kappa" distribution, hence their density is given by $[37,38]$ :

$$
n_{e}=n_{e, 0}\left(1-\frac{e \Phi}{T_{e}\left(\kappa-\frac{3}{2}\right)}\right)^{\left(-\kappa+\frac{1}{2}\right)}
$$




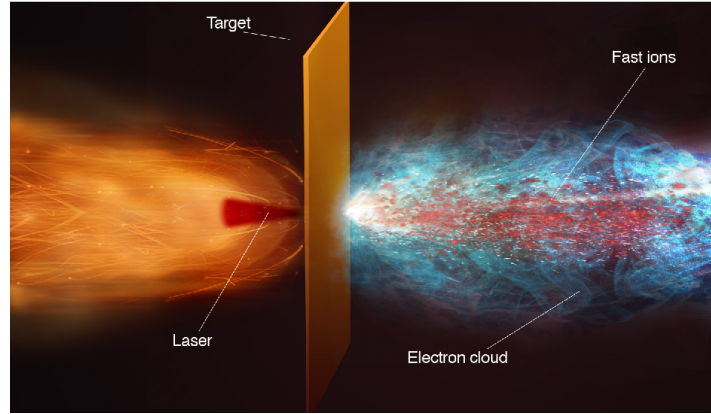

(a)

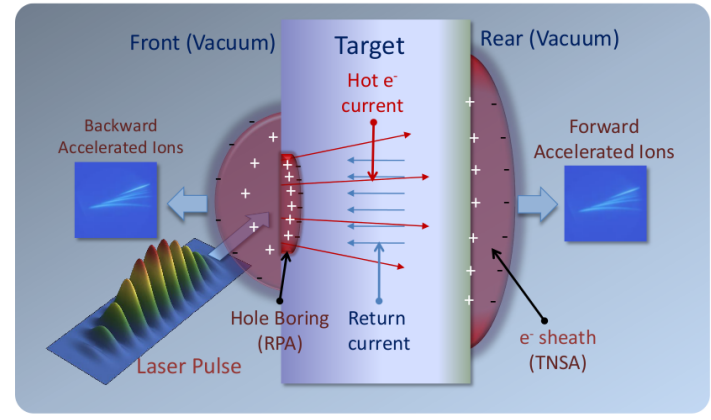

(b)

FIG. 1: (Color online) (a) The interaction of a laser beam with a solid target is illustrated; note that emission of fast ions from the rear surface of the target. (b) Ion acceleration mechanism (heuristic plot) due to laser-target interaction. Both plots have been adapted from Ref. 8, with permission from the publisher. where $\Phi$ is the electrostatic potential, viz. $E=-\partial \Phi / \partial x$. Here, $\kappa$ is the (real) spectral index which measures the strength of the excess superthermality, $T_{e}$ denotes electron temperature, and $e$ is the electron charge. Note that $\kappa>3 / 2$ is assumed as a requirement [37, 38].

The neutrality assumption (plasma approximation) is adopted, for analytical tractability. We shall assume throughout this work that the density gradient scale length is much greater than the Debye length, so that quasineutrality remains valid during the expansion procedure. Having adopted the plasma hypothesis, the Poisson equation for the electrostatic potential can be replaced by the relation (neutrality condition):

$$
n_{e}=z_{1} n_{1}+z_{2} n_{2}
$$

assumed to hold at all times.

\section{A. Electric field in a kappa-distributed electron background}

Upon inspection of the above equations, it is obvious that the only interaction between the two ion species is through the self-consistent electric field, entering the momentum equation for the ions. We thus need to evaluate the electric field, in order to close the system of evolution equations. Differentiating Eq. (2) with respect to $x$, and using the quasineutrality relation (3), we obtain

$$
\frac{\partial n_{e}}{\partial x}=\frac{\partial}{\partial x}\left(z_{1} n_{1}+z_{2} n_{2}\right)=\frac{e}{T_{e}} \frac{\left(\kappa-\frac{1}{2}\right)}{\left(\kappa-\frac{3}{2}\right)}\left(1-\frac{e \Phi}{T_{e}\left(\kappa-\frac{3}{2}\right)}\right)^{-1}\left(z_{1} n_{1}+z_{2} n_{2}\right) \frac{\partial \Phi}{\partial x}
$$

The electric field $E$ is thus determined by using Eq. (4) as

$$
E=-\frac{\partial \Phi}{\partial x}=-\frac{T_{e}}{e} \frac{1}{z_{1} n_{1}+z_{2} n_{2}}\left(\frac{\kappa-\frac{3}{2}}{\kappa-\frac{1}{2}}\right)\left(1-\frac{e \Phi}{T_{e}\left(\kappa-\frac{3}{2}\right)}\right) \frac{\partial}{\partial x}\left(z_{1} n_{1}+z_{2} n_{2}\right)
$$

The latter equation (5) generalizes the well known relation:

$$
e E=-\frac{T_{e}}{n_{e}} \frac{\partial n_{e}}{\partial x}
$$

for isothermal (inertialess) electrons obeying the Maxwell-Boltzmann distribution [58]. As expected, Eq.
(6) is recovered from Eq. (5) in the limit $\kappa \rightarrow \infty$. We shall assume adiabatic ion motion, which implies

$$
\frac{\partial P_{j}}{\partial x}=\frac{3 T_{j, 0}}{n_{j, 0}^{2}} n_{j}^{2} \frac{\partial n_{j}}{\partial x} \quad(\text { for } j=1,2)
$$


where index zero denotes values at the initial time $t=0$.

Combining Eqs. (1) - (7), we obtain

$$
\begin{aligned}
\frac{\partial n_{j}}{\partial t}+\frac{\partial}{\partial x}\left(n_{j} u_{j}\right) & =0 \\
\frac{\partial u_{j}}{\partial t}+u_{j} \frac{\partial u_{j}}{\partial x} & =-\frac{3 T_{j, 0}}{m_{j}} \frac{n_{j}}{n_{j o}^{2}} \frac{\partial n_{j}}{\partial x}-\frac{z_{j} T_{e}}{m_{j}} \frac{1}{z_{1} n_{1}+z_{2} n_{2}}\left(\frac{\kappa-\frac{3}{2}}{\kappa-\frac{1}{2}}\right)\left(1-\frac{e \Phi}{T_{e}\left(\kappa-\frac{3}{2}\right)}\right) \frac{\partial}{\partial x}\left(z_{1} n_{1}+z_{2} n_{2}\right) .
\end{aligned}
$$

Note that the neutrality assumption (3) was adopted in the last step, to eliminate the electron density.

\section{B. Scaling}

For analytical convenience, we shall now introduce normalized variables, according to the following scaling:

$$
\begin{gathered}
T=\omega_{p, 1} t, \quad X=\frac{x}{\lambda_{D i}}, \quad N_{j}=\frac{n_{j}}{n_{j 0}}, \\
V_{j}=\frac{u_{j}}{c_{s}} \quad \text { and } \quad \phi=\frac{e \Phi}{T_{e}} .
\end{gathered}
$$

Here, the time $(T)$ and space $(X)$ variables are respectively normalized by the (ion) plasma period $\omega_{p, 1}^{-1}=$ $\left(4 \pi e^{2} n_{1 o} z_{1}^{2} / m_{1}\right)^{-1 / 2}$ and the (ion) Debye length $\lambda_{D, 1}=$ $\left(4 \pi z_{1} e^{2} n_{1 o} / k_{B} T_{e}\right)^{1 / 2}$; the number density $N_{j}(j=$ $e, 1,2)$ and the velocity $V_{j}$ are normalized by the unperturbed number density $n_{j 0}$ and ion acoustic speed $c_{s}=\left(z_{1} k_{B} T_{e} / m_{1}\right)^{1 / 2}$; finally, the electrostatic potential $\phi$ is normalized by $k_{B} T_{e} / e$, where $T_{e}$ denotes the electron temperature, and $e$ is the elementary (electron) charge. Furthermore, the following dimensionless parameters are defined:

$$
\delta=\frac{z_{2} n_{20}}{z_{1} n_{10}}, \quad \gamma=\frac{q_{2} / m_{2}}{q_{1} / m_{1}}=\frac{z_{2} m_{1}}{z_{1} m_{2}} .
$$

The physical meaning of these parameters is transparent, as they respectively represent the charge density ratio and the specific charge (charge-to-mass) ratio, between the two ion populations. Note that $\delta \rightarrow 0$ (and $\gamma$ becomes irrelevant) in the vanishing "secondary-ion" limit $\left(n_{2}=\right.$ $0)$.

Applying the above scaling, Eqs. (1) are cast in the dimensionless form:

$$
\begin{aligned}
\frac{\partial N_{1}}{\partial T}+\frac{\partial}{\partial X}\left(N_{1} V_{1}\right) & =0, \\
\frac{\partial V_{1}}{\partial T}+V_{1} \frac{\partial V_{1}}{\partial X} & =-\frac{\partial \phi}{\partial X}-\alpha_{1} N_{1} \frac{\partial N_{1}}{\partial X}, \\
\frac{\partial N_{2}}{\partial T}+\frac{\partial}{\partial X}\left(N_{2} V_{2}\right) & =0, \\
\frac{\partial V_{2}}{\partial T}+V_{2} \frac{\partial V_{2}}{\partial X} & =-\gamma \frac{\partial \phi}{\partial X}-\alpha_{2} N_{2} \frac{\partial N_{2}}{\partial X}, \\
\frac{\partial^{2} \phi}{\partial X^{2}} & =(1+\delta) n_{e}-N_{1}-\delta N_{2},
\end{aligned}
$$

We have defined the quantity:

$$
\alpha_{j}=\frac{3}{z_{1}} \frac{T_{j, 0}}{T_{e}} \frac{n_{10}^{2}}{n_{j, 0}^{2}} \frac{m_{1}}{m_{j}},
$$

i.e., essentially,

$$
\alpha_{1}=3 \frac{T_{1,0}}{z_{1} T_{e}} \quad \text { and } \quad \alpha_{2}=\frac{3}{z_{1}} \frac{T_{2,0}}{T_{e}} \frac{n_{10}^{2}}{n_{2,0}^{2}} \frac{m_{1}}{m_{2}},
$$

adopting the notation in Ref. 58. Finally, we have defined the kappa-related parameters:

$$
a=\kappa-\frac{3}{2} \quad \text { and } \quad b=\kappa-\frac{1}{2} \text {. }
$$

The procedure adopted above in order to determine the electric field $E$ - see Eqs. (4)-(6) - may now be adapted to the rescaled (dimensionless) formulation of the problem. In dimensionless form, Eq. (4) becomes:

$$
\frac{1}{n_{e}} \frac{\partial n_{e}}{\partial X}=\frac{b}{a}\left(1-\frac{\phi}{a}\right)^{-1} \frac{\partial \phi}{\partial X}
$$

Thus the electric field becomes:

$$
E=-\frac{\partial \phi}{\partial X}=-\left(\frac{a}{b}\right)\left(1-\frac{\phi}{a}\right) \frac{1}{n_{e}} \frac{\partial n_{e}}{\partial X} .
$$

By using the neutrality assumption

$$
n e=\frac{1}{1+\delta}\left(N_{1}+\delta N_{2}\right)
$$

one obtains

$$
E=-\left(\frac{a}{b}\right)\left(1-\frac{\phi}{a}\right) \frac{1}{N_{1}+\delta N_{2}} \frac{\partial}{\partial X}\left(N_{1}+\delta N_{2}\right)
$$


By using Eq.(20) with Eqs.(11-15), we get

$$
\begin{aligned}
\frac{\partial N_{1}}{\partial T}+\frac{\partial}{\partial X}\left(N_{1} V_{1}\right) & =0 \\
\frac{\partial V_{1}}{\partial T}+V_{1} \frac{\partial V_{1}}{\partial X} & =-\alpha_{1} N_{1} \frac{\partial N_{1}}{\partial X} \\
& -\left(\frac{a}{b}\right)\left(1-\frac{\phi}{a}\right) \frac{\partial}{\partial X}\left[\ln \left(N_{1}+\delta N_{2}\right)\right], \\
\frac{\partial N_{2}}{\partial T}+\frac{\partial}{\partial X}\left(N_{2} V_{2}\right) & =0, \\
\frac{\partial V_{2}}{\partial T}+V_{2} \frac{\partial V_{2}}{\partial X} & =-\alpha_{2} N_{2} \frac{\partial N_{2}}{\partial X} \\
& -\gamma\left(\frac{a}{b}\right)\left(1-\frac{\phi}{a}\right) \frac{\partial}{\partial X}\left[\ln \left(N_{1}+\delta N_{2}\right)\right] .
\end{aligned}
$$

\section{SELF-SIMILAR EXPANSION SCHEME}

The system of partial differential equations (PDEs) (21) may now be transformed into a set of ordinary differential equations (ODEs) by assuming that all depen-

$$
\left(\begin{array}{cccc}
V_{1}-\xi & \alpha_{1} N_{1}+\frac{a}{b}\left(1-\frac{\phi}{a}\right) \frac{1}{N} & 0 & \left(\frac{a}{b}\right)\left(1-\frac{\phi}{a}\right) \frac{\delta}{N} \\
N_{1} & V_{1}-\xi & 0 & 0 \\
0 & \gamma \frac{a}{b}\left(1-\frac{\phi}{a}\right) \frac{1}{N} & V_{2}-\xi & \alpha_{2} N_{2}+\gamma \frac{a}{b}\left(1-\frac{\phi}{a}\right) \frac{\delta}{N} \\
0 & 0 & N_{2} & V_{2}-\xi
\end{array}\right)\left(\begin{array}{c}
V_{1}^{\prime} \\
N_{1}^{\prime} \\
V_{2}^{\prime} \\
N_{2}^{\prime}
\end{array}\right)=\mathbf{0}
$$

where prime denotes differentiation with respect to $\xi$. As boundary condition, we require that there should exist a point $\xi_{0}$ such that

$$
\begin{aligned}
V_{1}\left(\xi_{0}\right) & =0 & V_{2}\left(\xi_{0}\right) & =0, \\
N_{1}\left(\xi_{0}\right) & =1 & N_{2}\left(\xi_{0}\right) & =\delta .
\end{aligned}
$$

Eq. (22) can be written in matrix form as

Eqs. (22) reduce to

$$
\begin{aligned}
\left(V_{1}-\xi\right) \frac{N_{1}^{\prime}}{N_{1}}+V_{1}^{\prime} & =0 \\
\frac{a}{b}\left(1-\frac{\phi}{a}\right) \frac{N_{1}^{\prime}}{N_{1}}+\left(V_{1}-\xi\right) V_{1}^{\prime} & =0 .
\end{aligned}
$$

At this stage, the derivative terms in Eqs. (25) may be treated as independent variables. Considering the resulting set of algebraic equations, the determinant of the system must vanish, for nontrivial solutions to exist:

$$
\left|\begin{array}{cc}
V_{1}-\xi & 1 \\
\frac{a}{b}\left(1-\frac{\phi}{a}\right) & V_{1}-\xi
\end{array}\right|=0 .
$$

We choose the positive solution, corresponding to an expansion in the $+x$ direction and a velocity increasing with increasing $x$, so the solution of the determinant is

$$
V_{1}=\xi+\sqrt{\left(\frac{a}{b}\right)\left(1-\frac{\phi}{a}\right)} .
$$
light ions (protons) only and the ions are initially cold. So, in the single $\left(N_{2}=V_{2}=0\right)$ cold $\left(\alpha_{1}=0\right)$ ion case,

dent variables are functions of the similarity parameter

$$
\begin{aligned}
\left(V_{1}-\xi\right) N_{1}^{\prime}+N_{1} V_{1}^{\prime} & =0 \\
\left(V_{1}-\xi\right) V_{1}^{\prime} & =-\alpha_{1} N_{1} N_{1}^{\prime} \\
& -\left(\frac{a}{b}\right)\left(1-\frac{\phi}{a}\right)\left[\ln \left(N_{1}+\delta N_{2}\right)\right]^{\prime} \\
\left(V_{2}-\xi\right) N_{2}^{\prime}+N_{2} V_{2}^{\prime} & =0 \\
\left(V_{2}-\xi\right) V_{2}^{\prime} & =-\alpha_{2} N_{2} N_{2}^{\prime} \\
& -\gamma\left(\frac{a}{b}\right)\left(1-\frac{\phi}{a}\right)\left[\ln \left(N_{1}+\delta N_{2}\right)\right]^{\prime}
\end{aligned}
$$

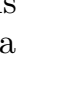


With the boundary condition $(23)$, we obtain $\xi_{0}=-\sqrt{\frac{a}{b}}$, i.e.,

$$
\xi_{0}=-\left(\frac{2 \kappa-3}{2 \kappa-1}\right)^{1 / 2}
$$

Interestingly, this relates the speed to the sound speed, since the ratio $a / b=(2 \kappa-3) /(2 \kappa-1)$ is essentially related to the sound speed (squared) for non-Maxwellian plasmas within the kappa-distribution approach [37, 38, 47] (see that $a / b \rightarrow 1$ in the infinite $\kappa$, i.e. Maxwellian limit).

Combining Eqs. (25) and (27), we find

$$
\frac{d \phi}{d \xi}=\frac{2 b}{1-2 b} \sqrt{\frac{1}{b}(a-\phi)} .
$$

One can derive a self-similar solution for the system under the assumption of charge quasi-neutrality, for the potential, the ion fluid speed, and for the ion density. The set of analytical expressions for the state variables thus obtained read:

$$
\begin{aligned}
\phi^{(s s)} & =\frac{-1}{(1-2 b)^{2}}\left[b\left(\xi-\xi_{0}\right)\left(2 \sqrt{\frac{a}{b}}(2 b-1)+\xi-\xi_{0}\right)\right], \\
V_{1} & =\xi+\sqrt{\frac{a}{b}\left(1-\frac{\phi^{(s s)}}{a}\right)}, \\
N_{1} & =\left(1-\frac{\phi^{(s s)}}{a}\right)^{-b},
\end{aligned}
$$

In the limit $(\kappa \rightarrow \infty)$, i.e, for a Maxwellian distribution, one readily recovers

$$
\begin{aligned}
\phi^{(s s)} & =-(\xi+1), \\
V_{1} & =\xi+1, \\
N_{1} & =\exp [-(\xi+1)]=\exp \left(-V_{1}\right) .
\end{aligned}
$$

These expressions are identical to those obtained earlier for electron-ion plasma $[23,24,58]$ considering Maxwellian electrons.

We can see from Eq. (31) that the density profile exponentially decreases, extending to infinity into vacuum, thus representing a rarefaction wave which propagates into the unperturbed plasma with velocity $c_{s}$, that is, the ion acoustic speed. The velocity increases linearly from zero at $\xi=-1$ to attain a constant value $\left(c_{s}\right)$ at $\xi=0$. In our case, Eq. (30) with superthermal effect through $\kappa$, we can see the velocity increase linearly from zero at $\xi=\xi_{0}$ (the rarefaction wave) and with $c^{s,(\kappa)}=\frac{2}{1-2 b} \sqrt{a b} c_{s}$ at $\xi=0$, where $c^{s,(\kappa)}$ is the modified ion acoustic speed in terms of $\kappa$.

The influence of superthermality on the ion density, velocity and the electric field is depicted in Fig. 2. We can see that ion acceleration is enhanced due to an excess in superthermal electrons, i.e. upon decreasing $\kappa$.

\section{B. Single warm-ion model}

Retaining the thermal pressure term in eq. (22), the single ion model equations read:

$$
\begin{aligned}
\left(V_{1}-\xi\right) \frac{N_{1}^{\prime}}{N_{1}}+V_{1}^{\prime} & =0 \\
{\left[\alpha_{1} N_{1}^{2}+\left(\frac{a}{b}\right)\left(1-\frac{\phi}{a}\right)\right] \frac{N_{1}^{\prime}}{N_{1}}+\left(V_{1}-\xi\right) V_{1}^{\prime} } & =0 .
\end{aligned}
$$

The compatibility (vanishing determinant) condition now becomes

$$
\left|\begin{array}{cc}
V_{1}-\xi & 1 \\
\alpha_{1} N_{1}^{2}+\left(\frac{a}{b}\right)\left(1-\frac{\phi}{a}\right) & V_{1}-\xi
\end{array}\right|=0
$$

which implies

$$
V_{1}=\xi+\sqrt{\frac{1}{b}\left[a-\phi+b \alpha_{1}\left(1-\frac{\phi}{a}\right)^{-2 b}\right]} .
$$

Requiring $V_{1}=0$ and $\phi=0$ at $\xi=\xi_{0}$, we obtain $\xi_{0}=$ $-\sqrt{\frac{a+b \alpha_{1}}{b}}$.

For $\kappa \rightarrow \infty$ i.e, for a Maxwellian electron distribution, we recover [58]:

$$
V_{1}=\xi+\sqrt{1+\alpha_{1} \exp (2 \phi)}
$$

Combining Eqs. (32) and (34) with the neutrality condition $z_{1} N_{1}=n_{e}$, we find that the electrostatic potential is given by the equation

$$
\frac{d \phi}{d \xi}=-\frac{2 a b\left(1-\frac{\phi}{a}\right)^{2 b+\frac{3}{2}} \sqrt{\frac{a}{b}+\alpha_{1}\left(1-\frac{\phi}{a}\right)^{-(2 b+1)}}}{4 b^{2} \alpha_{1}+a(2 b-1)\left(1-\frac{\phi}{a}\right)^{2 b+1}} .
$$

The electrostatic potential, ion velocity and ion density are obtained numerically upon solving Eqs. (32) and (36) numerically. These results are shown in Fig. 3 and 4 .

Fig. 4 shows the effect of the ionic thermal pressure on ion acceleration. Upon increasing the ion temperature, the velocity of the expansion front increases, suggesting that thermal effects energize the ions, as intuitively expected.

\section{Two cold-ion species plasma: the role of minority ions}

Here, we consider that in addition to the main ions $N_{1}$, of mass $m_{1}$ and charge $z_{1}$, the plasma slab contains a small admixture of ions of mass $m_{2}$ and charge $z_{2}$. Accordingly, the variation of the second fluid is assumed to be slow, viz. $N_{2}^{\prime} \ll N_{1}^{\prime}$ [64]. Taking $N_{2} \ll N_{1}$, Eq. 


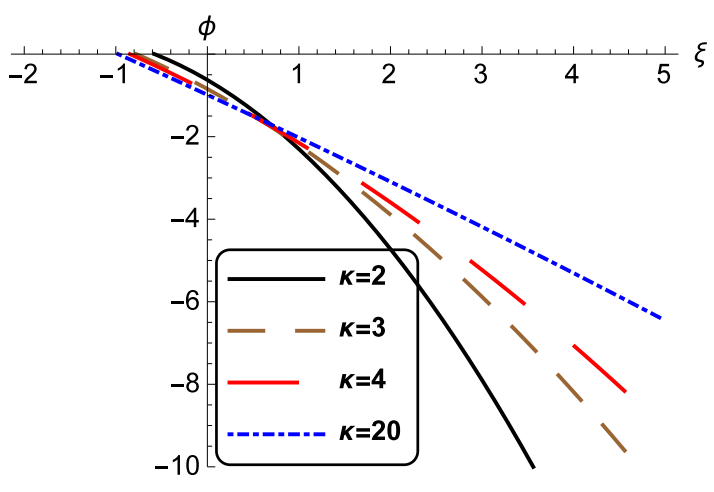

(a)

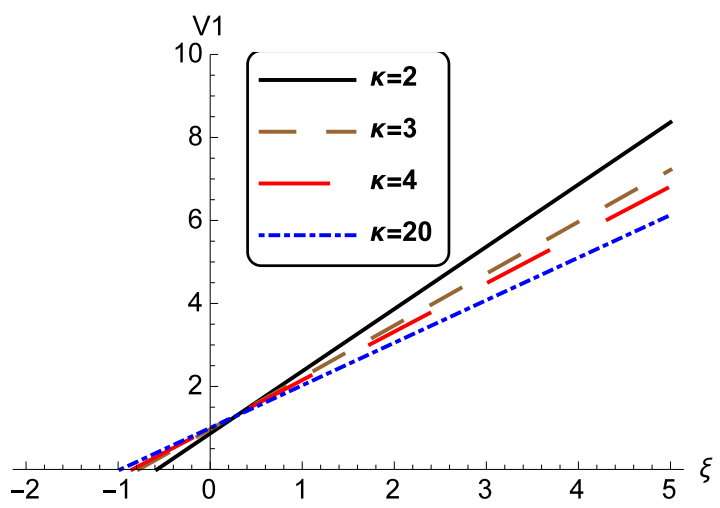

(c)

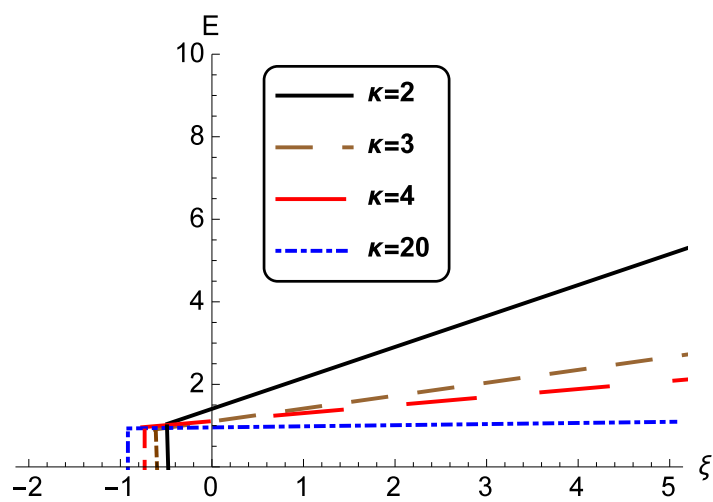

(b)

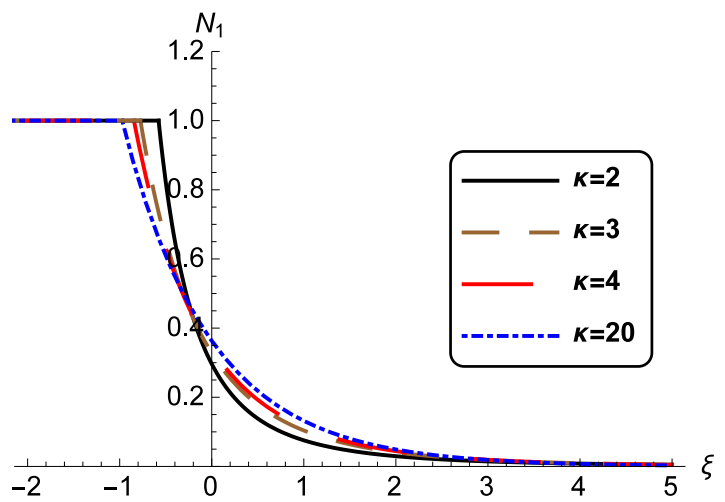

(d)

FIG. 2: (Color online) Single cold ion fluid model: the state variables ((a) electrostatic potential (b) electric field (c) ion fluid speed and (d) density) are depicted versus $\xi$, following (30). Larger values of $\kappa$ recover the known Maxwellian result [23, 24, 58].

(24) decouples (neglecting partial pressure $\alpha_{1}=\alpha_{2}=0$ )

$$
\begin{aligned}
\left(V_{1}-\xi\right) N_{1}^{\prime}+N_{1} V_{1}^{\prime} & =0 \\
\left(V_{1}-\xi\right) V_{1}^{\prime} & \simeq-\frac{a}{b}\left(1-\frac{\phi}{a}\right)\left(\ln N_{1}\right)^{\prime}, \\
\left(V_{2}-\xi\right) N_{2}^{\prime}+N_{2} V_{2}^{\prime} & =0 \\
\left(V_{2}-\xi\right) V_{2}^{\prime} & \simeq-\gamma \frac{a}{b}\left(1-\frac{\phi}{a}\right)\left(\ln N_{1}\right)^{\prime} .
\end{aligned}
$$

or, in matrix form:

$$
\left(\begin{array}{cccc}
V_{1}-\xi & \frac{a}{b}\left(1-\frac{\phi}{a}\right) \frac{1}{N_{1}} & 0 & 0 \\
N_{1} & V_{1}-\xi & 0 & 0 \\
0 & \gamma \frac{a}{b}\left(1-\frac{\phi}{a}\right) \frac{1}{N_{1}} & V_{2}-\xi & 0 \\
0 & 0 & N_{2} & V_{2}-\xi
\end{array}\right)\left(\begin{array}{c}
V_{1}^{\prime} \\
N_{1}^{\prime} \\
V_{2}^{\prime} \\
N_{2}^{\prime}
\end{array}\right) \simeq \mathbf{0}
$$

(30), implying that

$$
\left(V_{2}-\xi\right) V_{2}^{\prime} \simeq-\gamma \frac{d \phi^{(s s)}}{d \xi}
$$

This is an approximate relation for $V_{2}$, in terms of the self-similar solution $\phi^{(s s)}$ that was given by Eq. (30).

The numerical results from the latter equations are depicted in Fig. 5. Note that the variables $\left(N_{1}\right.$ and $V_{1}$ ) corresponding to the (dominant) first ion fluid remain precisely as they were obtained in Subsection III B above, since Eq. (37) is still valid in this case (as discussed earlier in this Section) and it does not involve the second (minority ion) population $N_{2}$. However, the second (minority ion) population is affected by the first, as obvious in Eqs. (38) and (40) above.

It is worth mentioning that the "minority-ion" fluid assumptions $N_{2} \ll N_{1}$ and $N_{2}^{\prime} \ll N_{1}^{\prime}$ are a posteriori satisfied by the numerical values obtained for the density variables; see Fig. 5(c).

The solution of Eq. (37) is, once again, given by Eq. 


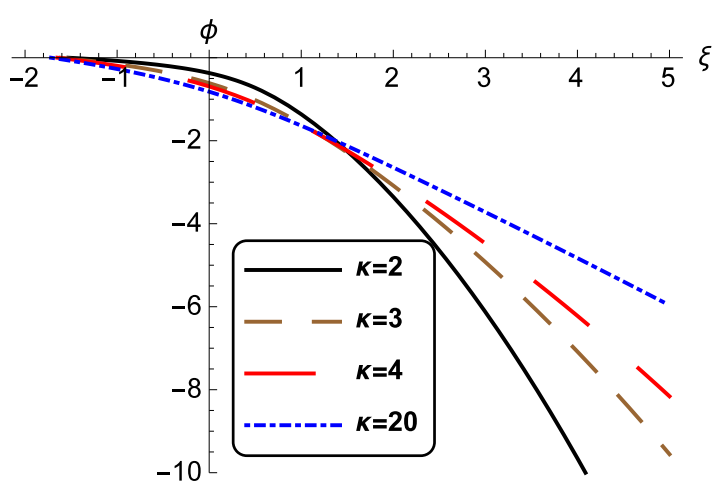

(a)

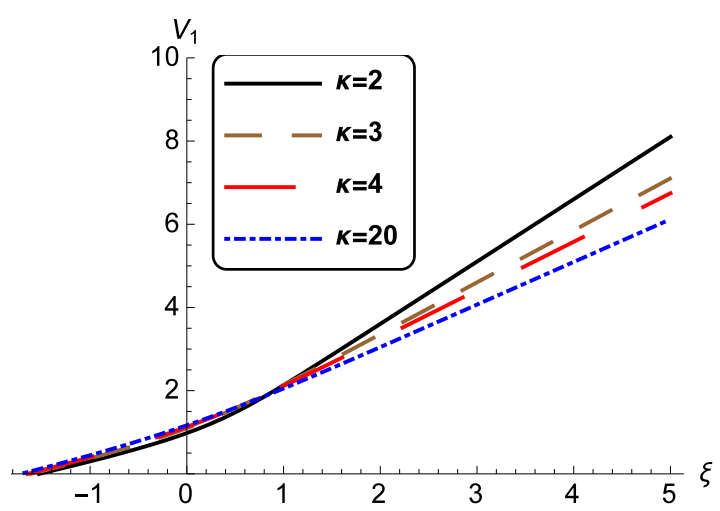

(c)

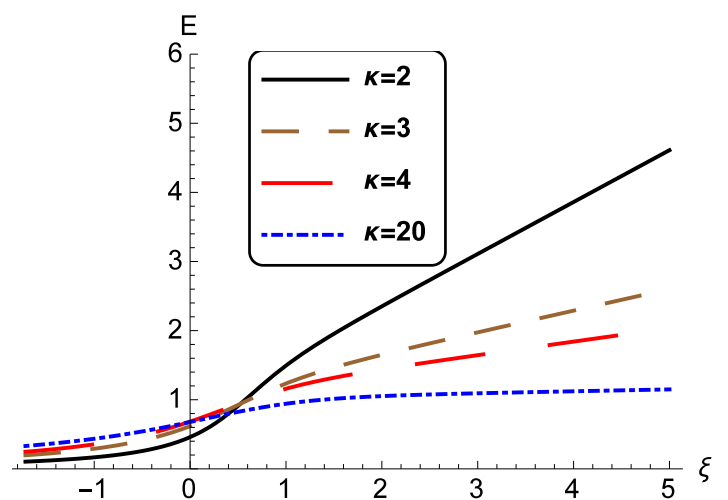

(b)

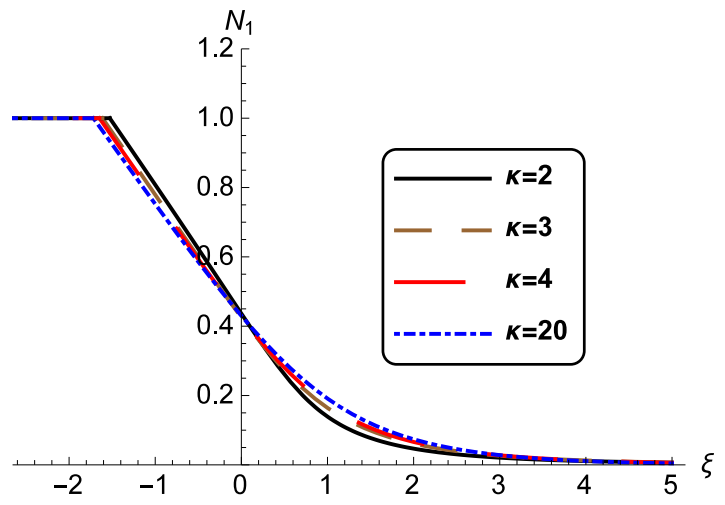

(d)

FIG. 3: (Color online) Single (warm) ion fluid model: the effect of superthermality is shown, following Eqs. (32) and (36). The state variables ((a) electrostatic potential (b)electric field (c) ion fluid speed and (d) density) are depicted versus $\xi$. We have considered $\alpha_{1}=1$, as an indicative value.

D. Two warm-ion model: the role of minority ions

Here, we consider the plasma slab is consisted of two warm ion species. Under the minority (second) ion assumption, viz. assuming $N_{2} \ll N_{1}$, Eq. (24) becomes:

$$
\begin{aligned}
\left(V_{1}-\xi\right) \frac{N_{1}^{\prime}}{N_{1}}+V_{1}^{\prime} & =0 \\
\left(V_{1}-\xi\right) V_{1}^{\prime} & \simeq-\alpha_{1} N_{1} N_{1}^{\prime}-\frac{a}{b}\left(1-\frac{\phi}{a}\right)\left(\ln \left[N_{1}\right]\right)^{\prime}
\end{aligned}
$$

$\left(V_{2}-\xi\right) N_{2}^{\prime}+N_{2} V_{2}^{\prime}=0$,

$$
\left(V_{2}-\xi\right) V_{2}^{\prime} \simeq-\alpha_{2} N_{2} N_{2}^{\prime}-\gamma \frac{a}{b}\left(1-\frac{\phi}{a}\right)\left(\ln \left[N_{1}\right]\right)^{\prime}
$$

or, in matrix form : 


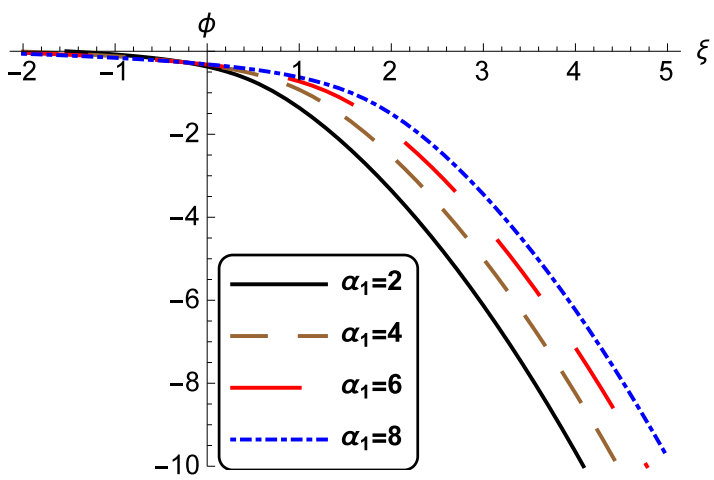

(a)

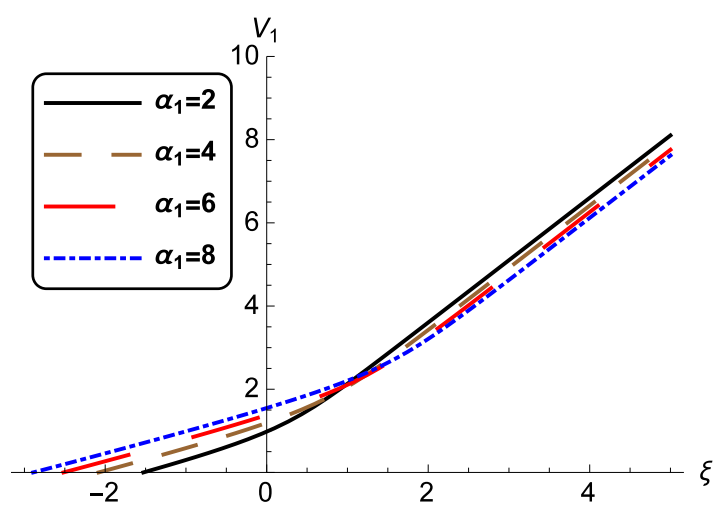

(c)

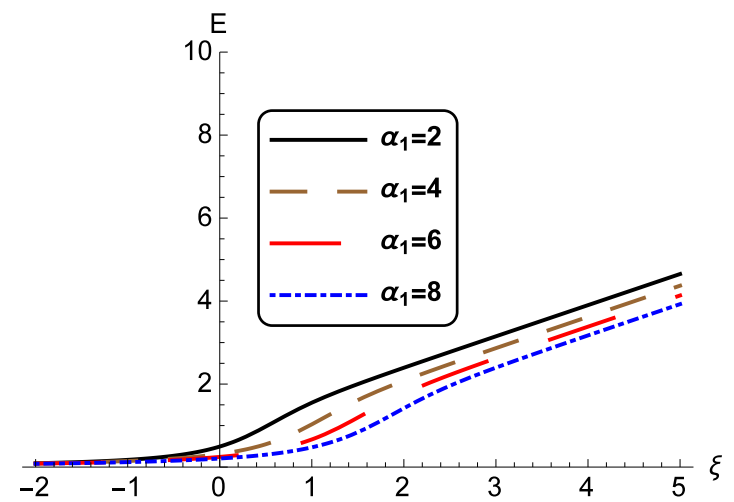

(b)

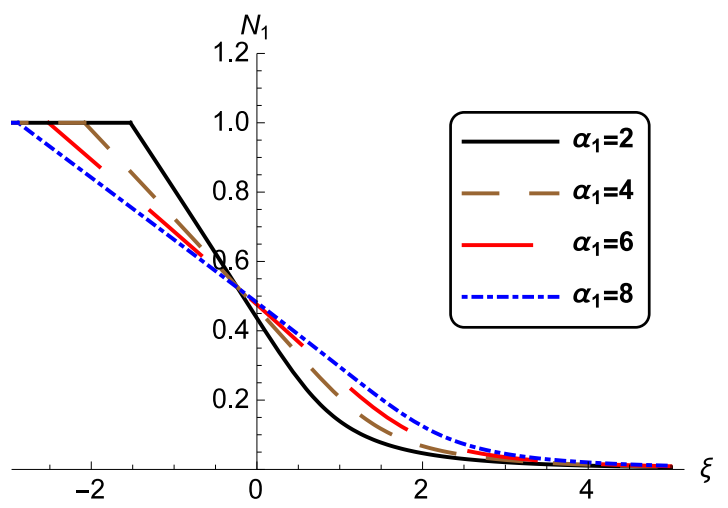

(d)

FIG. 4: (Color online) Single (warm) ion fluid model: the effect of thermal pressure is shown, following Eqs. (32) and (36). The state variables ( (a) electrostatic potential (b) electric field (c) ion fluid speed and (d) density) are depicted versus $\xi$. We have considered $\kappa=2$ as indicative value.

$$
\left(\begin{array}{cccc}
V_{1}-\xi & \alpha_{1} N_{1}+\frac{a}{b}\left(1-\frac{\phi}{a}\right) \frac{1}{N_{1}} & 0 & 0 \\
N_{1} & V_{1}-\xi & 0 & 0 \\
0 & \gamma \frac{a}{b}\left(1-\frac{\phi}{a}\right) \frac{1}{N_{1}} & V_{2}-\xi & \alpha_{2} N_{2} \\
0 & 0 & N_{2} & V_{2}-\xi
\end{array}\right)\left(\begin{array}{c}
V_{1}^{\prime} \\
N_{1}^{\prime} \\
V_{2}^{\prime} \\
N_{2}^{\prime}
\end{array}\right) \simeq \mathbf{0}
$$

The system of Eqs. (42) may be solved numerically. The results are shown in Figs. 6 for the minority (second) ion species. As in the cold-ion case above, the variables corresponding to the dominant first fluid remain as they were obtained in Subsection III B above, since Eq. (41) is not affected by the 2 nd population $N_{2}$.
E. Two warm-ion model: arbitrary ion admixture

For the sake of analytical tractability (and also, for comparison with Ref. 58), we have assumed above that the concentration of the secondary ions is much lower that that of the principal ion population, i.e. $\delta \ll 1$, hence the influence of the secondary ion (2) population on the motion of the main ion fluid was practically neg- 


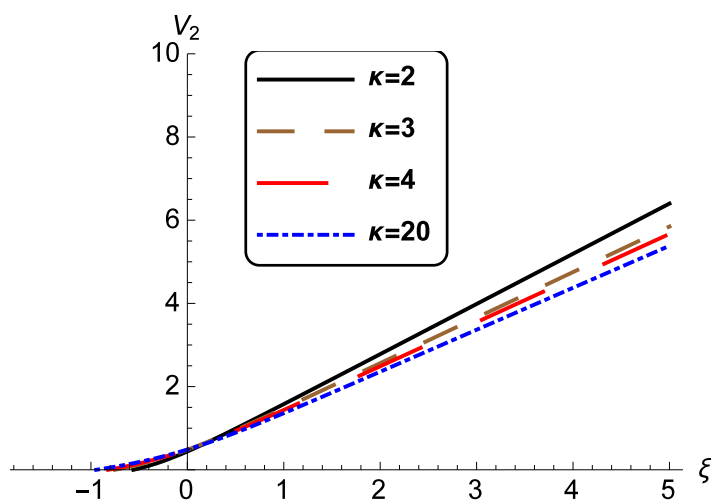

(a)

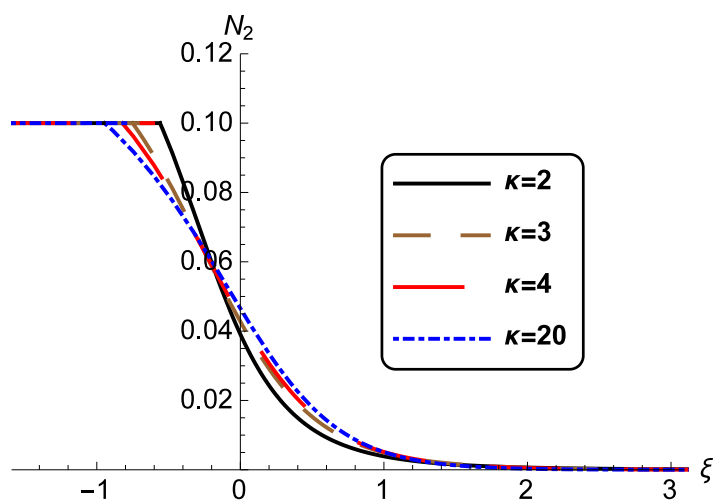

(b)

FIG. 5: (Color online) Two (cold) ion-fluid model, with minority ions $\left(N_{2} \ll N_{1}\right)$ : the effect of superthermality is shown, following Eqs. (40). (a) the second (minority) ion-fluid speed and (b) density are depicted, versus the self-similar space variable $\xi$ (taking $\alpha_{1}=\alpha_{2}=0$ and $\gamma=1 / 3$, i.e. $z_{1}=z_{2}$ and $m_{2}=3 m_{1}$ ). Note that the variables corresponding to the dominant first fluid are omitted, since they are exactly given in Figure 2 above, obtained from Eq. (37), in this case too.

ligible. Now, we turn to the full two-fluid problem where these concentrations may be comparable in order of magnitude, i.e., the plasma slab contains a mixture of two types of ions in an arbitrary proportion, manifested by a finite value of $\delta(<1)$. The expansion of the plasma slab into vacuum is described by the system of Eqs. (24). Considering all the derivatives $\left(V_{1}^{\prime}, N_{1}^{\prime}, V_{2}^{\prime}, N_{2}^{\prime}\right)$ as independent variables and the resulting set of equations as an algebraic (Cramer) system, the secular (vanishing determinant) condition resulting from Eqs. (24) may be cast in the form:

$$
\frac{\left(\frac{a}{b}\right)\left(1-\frac{\phi}{a}\right) \frac{N_{1}}{N}}{\left(V_{1}-\xi\right)^{2}-\alpha_{1} N_{1}^{2}}+\frac{\left(\frac{a}{b}\right)\left(1-\frac{\phi}{a}\right) \frac{\gamma \delta N_{2}}{N}}{\left(V_{2}-\xi\right)^{2}-\alpha_{2} N_{2}^{2}}=1 .
$$

This equation cannot be solved analytically to find $N_{1}, N_{2}, V_{1}, V_{2}$. However, it can be used to determine $\xi_{0}$ as the negative root of a polynomial equation, which corresponds to an expansion in the $+x$ direction and a velocity increasing with increasing $x$. Assuming the initial condition (23) above, it is straightforward to show that Eq. (44) leads to:

$$
\xi_{0}^{4}+A \xi_{0}^{2}+B=0
$$

viz.,

$$
\xi_{0}=-\left[\frac{-A+\sqrt{A^{2}-4 B}}{2}\right]^{1 / 2}
$$

where $A=-\left[a(1+\gamma \delta)+b\left(\alpha_{1}+\alpha_{2}\right)(1+\delta)\right] /[b(1+\delta)]$ and $B=b \alpha_{1} \alpha_{2}(1+\delta)+a\left(\alpha_{2}+\alpha_{1} \gamma \delta\right)$. The negative sign in the latter expression for $\xi_{0}$ denotes the fact that the a rarefaction wave moves in the direction opposite to that of the expanding plasma. The modified speed of the rarefaction wave is $\xi_{0}$ (which essentially represents the sound speed in the presence of the two ion components). It may be appropriate to compare to the (far simpler) expression (28) above, which is indeed recovered in the limit $\delta=0$ with $\alpha_{1}=\alpha_{2}=0$.

In order to investigate the full problem, i.e. retaining the secondary ion fluid inertia, we have solved the system of Eqs. (24) numerically, taking into account the condition (44) and the initial value of the self-similar variable $\xi_{0}(46)$. The result of the numerical integrations is shown in Figs. 7 and 8, for the cold- $\left(\alpha_{1}=\alpha_{2}=0\right)$ and warm- $\left(\alpha_{1}=\alpha_{2}=1\right)$ ion case(s), respectively. The plots depicted in Fig. 7 actually represent the same set of variables (namely, $\gamma=1 / 3$ and $\alpha_{1}=\alpha_{2}=0$ ) as the one shown in Figs. 2 and 5, except for the fact that the assumption $N_{1} \ll N_{2}$ is now dropped, viz., a finite value of $N_{2}$ is assumed in Fig. 7 (where $\delta=1$ is taken, rather $\delta \ll 1$, as in Fig. 5. Similarly, the plots depicted in Fig. 8 represent the same set of variables (namely, $\gamma=1 / 3$ and $\alpha_{1}=\alpha_{2}=1$ ) as the one shown in Figs. 3 and 6 , except that a finite value of $N_{2}$ is assumed in Fig. 8 (i.e. $\delta=1$ ), rather $\delta \ll 1$, as in Fig. 6. In all of Figs. 2-8, we have considered a set of indicative (ad hoc) parameter values, namely $z_{2}=z_{1}$ and $m_{2}=3 m_{1}$, corresponding to $\gamma=1 / 3$ ( the values of $\delta$ are presented in the respective caption). We have solved the system numerically for many different values of the relevant parameters qualitatively same.

Upon a simple inspection of Figs. 2 and 5, on one hand, and Fig. 7 on the other, in comparison with one another, we see that, for different values of the superthermal spectral index $\kappa$, the electric field and the velocity of the ion species increases for higher values of the density ratio $\delta$. For large values of $\kappa$ (i.e. in the Maxwellian limit), Figs. 4 and 5 in Ref. [58] are exactly recovered, thus corroborating our results.

As shown in Fig. 7, for small values of $\kappa$, the number 


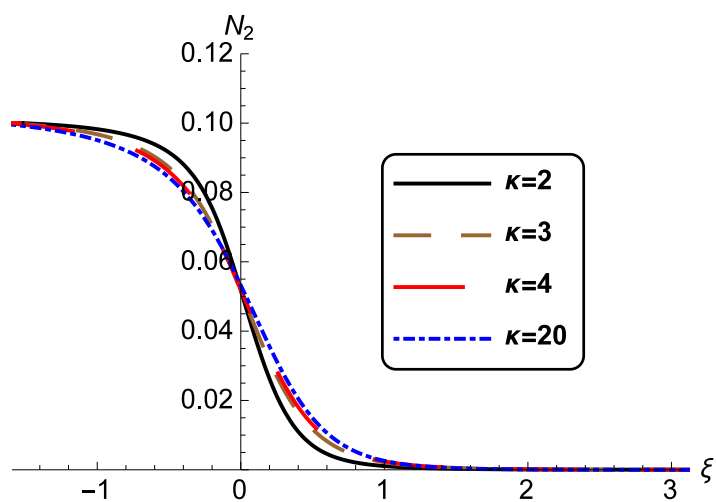

(a)

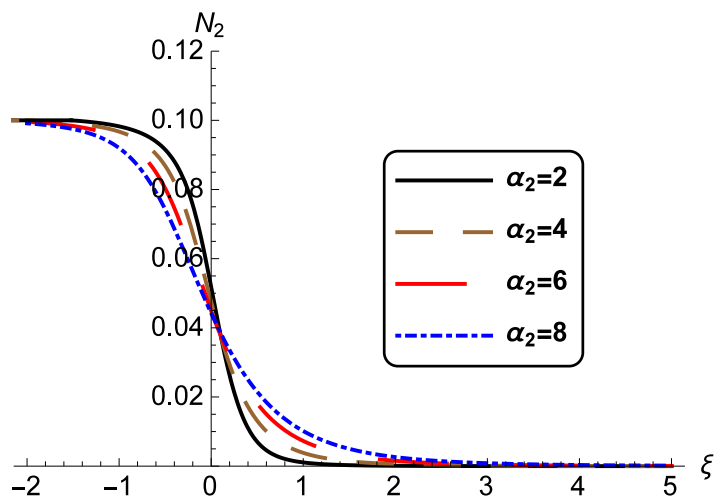

(c)

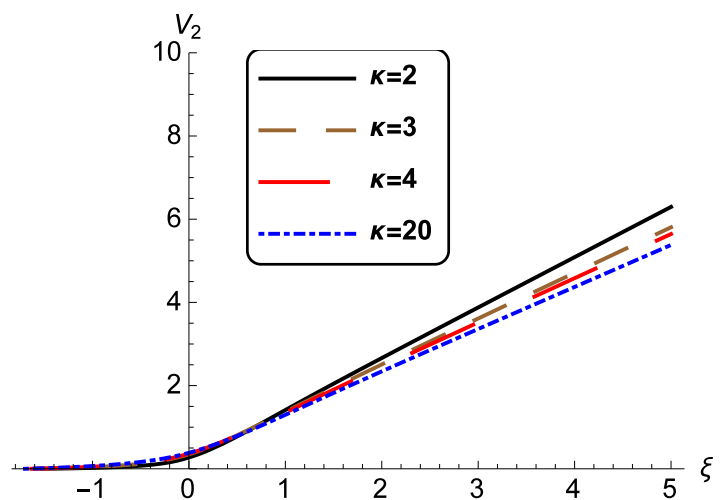

(b)

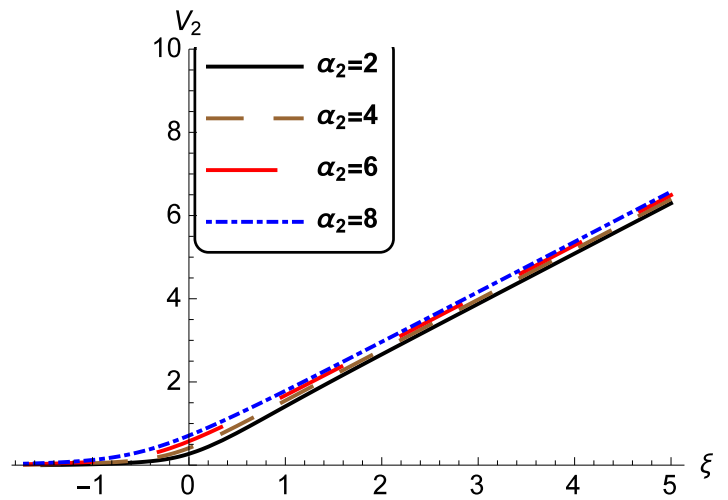

(d)

FIG. 6: (Color online) Minority ion 2-fluid model (warm): the effect of superthermality is shown, following Eqs. (42) (taking $\kappa=2, \alpha_{1}=1$ and $\gamma=1 / 3$, i.e. $z_{1}=z_{2}$ and $m_{1}=3 m_{2}$.

density $N_{1}$ and $N_{2}$ decrease sharply at the expansion front; considering thermal effects (in Fig.8) the transition is smoother.

We have also investigated the effect of the density ratio $\delta$ for two cases: $\gamma<1$ (Fig. 9) and $\gamma>1$ (see Fig. 10). Case 1: For $\gamma\left(=z_{2} m_{1} / z_{1} m_{2}\right)<1$, Figs. $9(\mathrm{a}, \mathrm{b})$ show that the electric field increases with increasing density ratio $\delta\left(=z_{2} n_{20} / z_{1} n_{10}\right)$. From Figs. 9(c,e) we can see that the concentration of the ions falls rapidly, attaining zero at some distance from the source plasma. Finally, Figs. $9(\mathrm{~d}, \mathrm{f})$ show that the velocity of both ions increase upon increasing $\delta$, i.e. for a stronger second ion concentration. Case 2: For $\gamma>1$, contrary to the previous case (1), we have considered a large value of $\gamma\left(\gamma=16\right.$, e.g. $z_{2}=$ $z_{1}$ and $\left.m_{1}=16 m_{2}\right)$, actually inspired by Ref. 60 . As physically expected, the 2nd ion inertia is dominant here. This is reflected in the plots in Figs. 10(a, b), which show that the electric field decreases upon increasing $\delta$. Fig.10(c, e) also shows that the concentration of both ions decreases slowly with increasing $\delta$. Furthermore, in Fig. 10(d, f) we see that the velocity now increases faster for higher $\delta$. This is intuitively expected, since the first ("principal") ion fluid accelerates the faster, during the expansion process, the heavier the secondary ions are $(\gamma>1)$, if the second ion fluid inertia is retained in the description.

As a final test, we have integrated numerically the full fluid equations for a small value of the ratio $N_{2} / N_{1}$ (= 0.1 ), in order to compare to the analytical solution obtained in Section $\mathrm{C}$ above (for $N_{2} \ll N_{1}$ ). The result, shown in Fig. 11, could not be more satisfactory: a simple comparison with Figs. 2 and 5 shows that all variables are practically identical, in all case, asides a small change in the density $N_{2}$ (due to the inertia of the 2nd fluid being retained: cf. Fig. 11 and 5. It therefore appears that the analytical result, obtained by neglecting the secondary ion inertia, succeeds in capturing the essential features of the expansion process, for small values of the density ratio $N_{2} / N_{1}$. As expected, larger values of $N_{2} / N_{1}$ (and of $M_{2} / M_{1}$ ) may lead to substantial deviation, however.

The latter considerations, based on Fig. 11, refer to the cold ion model, and to a given (arbitrary) set of pa- 
rameter values. We have repeated the same procedure (numerical integration) in a wide range of values, and are able to assert that the behavior described above is generic, i.e. valid throughout a wide range of parameters. (The corresponding plots are here omitted for brevity.)

\section{CONCLUSIONS}

Summarizing, we have established an analytical model to study the self similar expansion of semi infinite multispecies plasma slab into vacuum. We have taken into account the effect of the initial temperature of the plasma slab on the electrostatic field at the plasma slab and vacuum interface and on the acceleration of the escape ions from the slab. Our findings recover earlier results in the respective limits, in terms of the model parameter values.

We have also studied the effect of a non-Maxwellian electron distribution on the plasma expansion mechanism, in various cases. The expanding plasma front appears to be accelerated by the suprathermal electrons (i.e. for lower values of the superthermality index $\kappa$ ) more efficiently, as intuitively expected. For infinite $\kappa$, the Maxwellian limit is recovered.

Finally, we have studied the effect of the finite inertia of the second ion fluid, by integrating the exact system of equations numerically, for finite (non-negligible) values of the second-to-first ion density ratio $\delta$, and for different values of the relative charge-to-mass ratio $\gamma$. We have shown that including the second ion inertia modifies the analytical result only quantitatively (retaining the general qualitative characteristics of the expansion front), and even more so for heavier secondary ions, as physically expected.
The analytical model employed in our study certainly has its limitations and possible shortcomings. Clearly, the self similar solution is a special solution of the fluid equations and it does not allow for a detailed characterization of the entire process. The self similar solution is worthy and useful in its own merit for the description of the plasma expansion in a simple and analytically tractable way. Numerical simulations suggest that, with the passage of time, the expanding plasma profile approaches the form provided by the self-similar methodology $[25,34,62,63]$. Furthermore, we have assumed that the electron temperature remains constant throughout the plasma expansion process. This is adopted as an ad hoc assumption in our study, for the sake of tractability. This hypothesis may be questioned by meticulous interpretation of (e.g. PIC) plasma simulations. The latter, though imposed, goes beyond our scope in this study.

Our results are important in various physical situations involving plasma expansion, including ion acceleration applications for medical purposes, astrophysical situations and laser-assisted fusion schemes. PIC simulations [19-21] should be undertaken in the future, to corroborate our results.

\section{Acknowledgments}

Our work was supported financially by Queen's University (QUB), Centre for Plasma Physics (CPP). One of us (I.S. Elkamash) acknowledges financial support via an Egyptian Government fellowship. Dr Brian Reville (Queen's University Belfast) is warmly thanked, both for technical assistance and for a number of inspiring discussions.
[1] E. L. Clark, K. Krushelnick, M. Zepf, F. N. Beg, M. Tatarakis, A. Machacek, M. I. K. Santala, I. Watts, P. A. Norreys, and A. E. Dangor, Phys. Rev. Lett. 85, 1654 (2000).

[2] R. A. Snavely et al., Phys. Rev. Lett. 85, 2945 (2000).

[3] S. P. Hatchett et al., Phys. Plasmas 7, 2076 (2000).

[4] Laser-Plasma Interactions and Applications, Paul McKenna, David Neely, Robert Bingham and Dino Jaroszynski (Eds.) (Springer, 2013).

[5] A Superintense LaserPlasma Interaction Theory Primer, Andrea Macchi (Springer, 2013).

[6] M. Borghesi, A. J. Mackinnon, D. H. Campbell, D. G.Hicks, S. Kar, P. K. Patel, D. Price, , L. Romagnani, A. Schiavi, and O. Willi, Phys. Rev. Lett. 92, 055003 (2004).

[7] S. V. Bulanov, T. Z. Esirkepov, D. Habs, F. Pegoraro, and T. Tajima, Eur. Phys. J. D 55, 483 (2009).

[8] Andrea Macchi, Marco Borghesi and Matteo Passoni, Rev. Mod. Phys. 85, 751 (2013).

[9] S. Busold et al., Nucl. Instrum. Meth. Phys. Res. A 740, 94 (2014).

[10] N. Naumova , T. Schlegel, V. T. Tikhonchuk, , C.
Labaune, I. V. Sokolov, and G. Mourou, Phys. Rev. Lett. 102, 025002 Jan (2009).

[11] C. Regan, T. Schlegel, V. T.Tikhonchuk, J. J. Honrubia, J.L. Feugeas, and P. Nicola, Plasma Phys. Controlled Fusion 53, 045014 (2011).

[12] V.T.Tikhonchuk, A.Colatis, A.Vallet, E.Llor Aisa, G.Duchateau, Ph.Nicola and X.Ribeyre, Plasma Phys. Control. Fusion 58, 014018, (2015)

[13] Yunchao Zhang, Christine Charles, and Rod Boswell, Phys. Rev. Lett. 116, 025001 (2016).

[14] S. V. Bulanov, T. Zh. Esirkepov, V. S. Khoroshkov, A. V. Kuznetsov, and F. Pegoraro, Phys. Lett. A 299, 240 (2002).

[15] V. Malka, S. Fritzler, E. Lefebvre, E. dHumires, R. Ferrand, G. Grillon, C. Albaret, S. Meyroneinc, J.P. Chambaret, A. Antonetti, and D. Hulin, Med. Phys. 31, 1587 (2004).

[16] C. M. Ma, I. Veltchev, E. Fourkal, J. S. Li, W. Luo, , J. Fan, T. Lin, and A. Pollack, Laser Phys. 16, 639646 (2006).

[17] K.W.D. Ledingham,W.Galster, and R. Sauerbrey, Br.J.Radiol, 80, 855 (2007). 


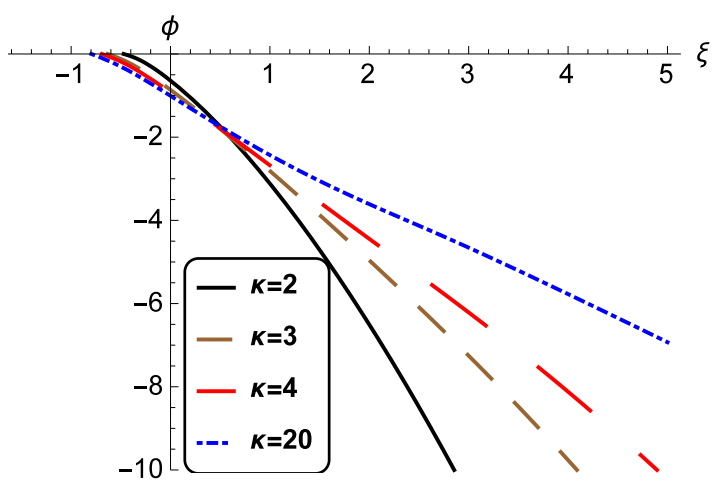

(a)

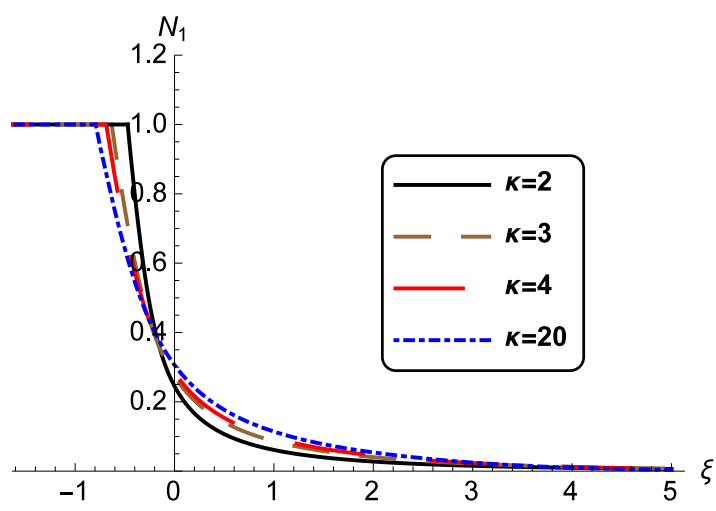

(c)

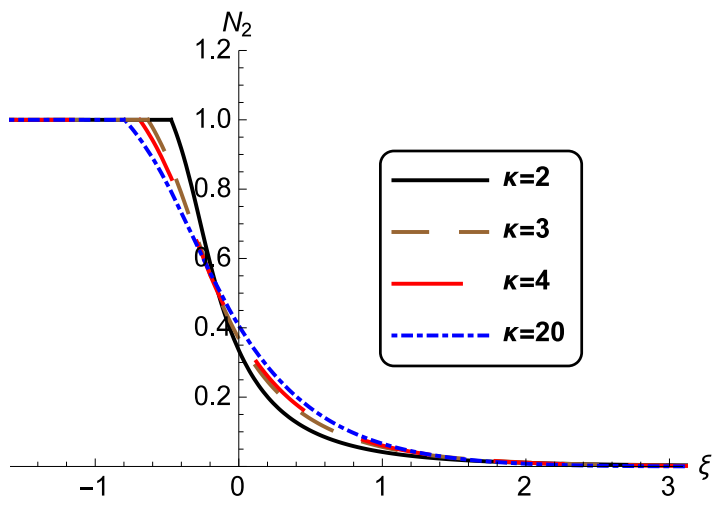

(e)

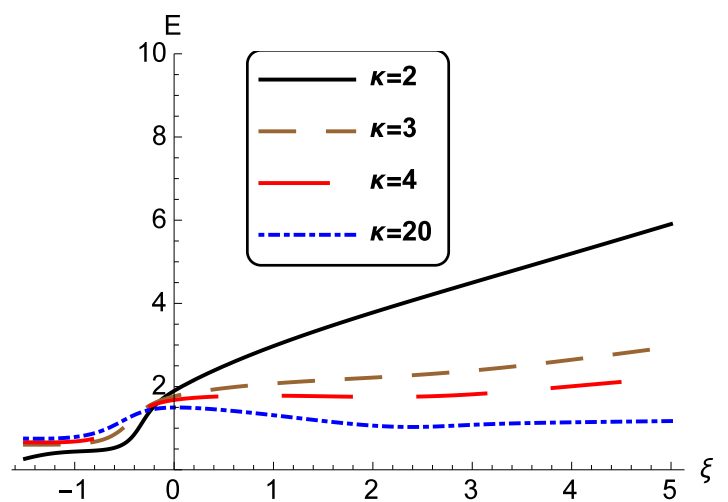

(b)

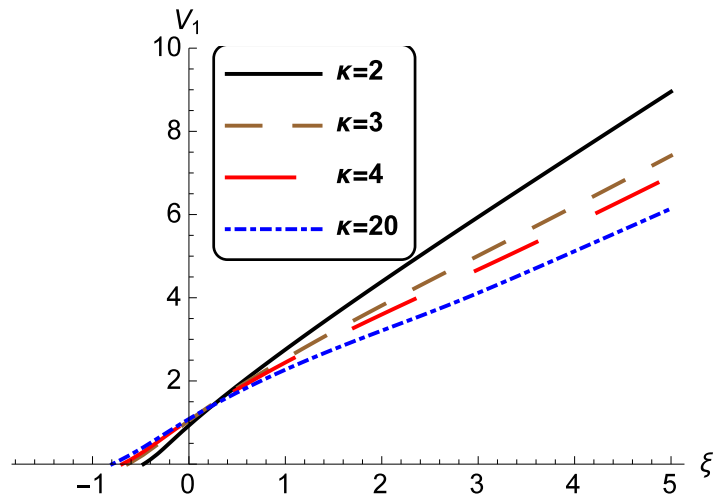

(d)

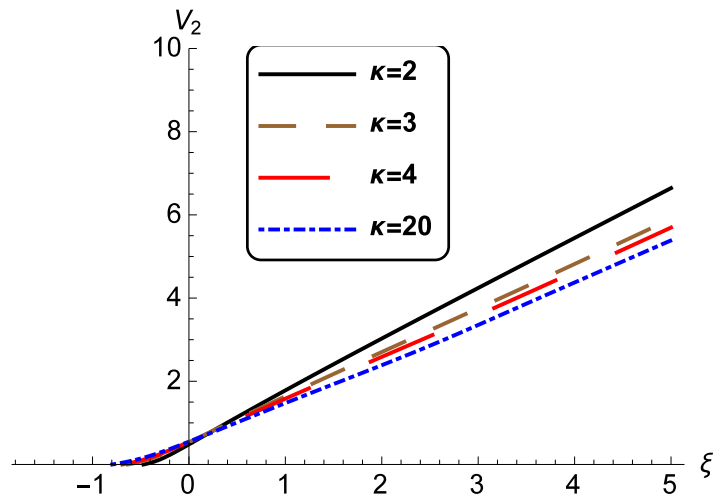

(f)

FIG. 7: (Color online) Electric field, electrostatic potential, density and velocity profiles of the two ion species with $\delta=1$, $\gamma=1 / 3$ and $\alpha_{1}=\alpha_{2}=0$, i.e. $z_{1}=z_{2}, m_{2}=3 m_{1}$ and $n_{20}=n_{10}$. Different values of $\kappa$ have been considered (see inset frame).

[18] S. D. Kraft et al., New J. Phys. 12, 085003 (2010).

[19] G. Sarri, M. E. Dieckmann, I. Kourakis, and M. Borghesi, Phys. Plasmas 17, 082305 (2010).

[20] G. Sarri, G. C. Murphy, M. E. Dieckmann, A. Bret, K. Quinn, I. Kourakis, M. Borghesi, L. O. C. Drury and A.
Ynnerman, New J. Phys 13, 073023/1-22 (2011).

[21] G. Sarri, M. E. Dieckmann, I. Kourakis, and M. Borghesi, Phys. Rev. Lett. 107, 025003 (2011).

[22] A.V. Gurevich, L.V. Pariiskaya, and L. P. Pitaevskii, Sov. Phys. JETP 22, 449 (1966). 


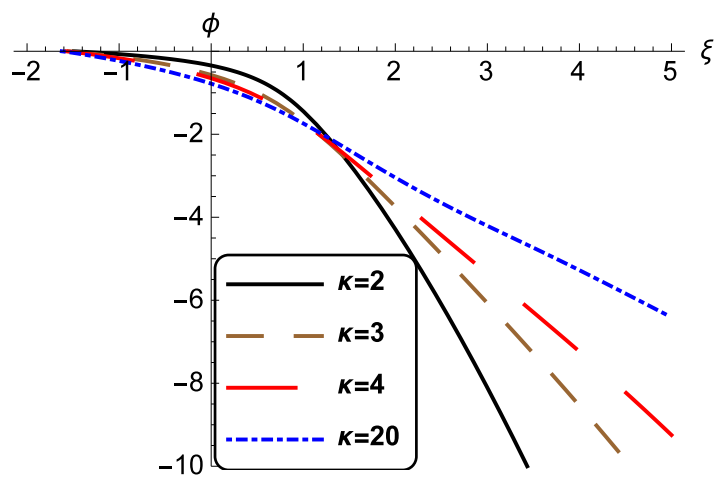

(a)

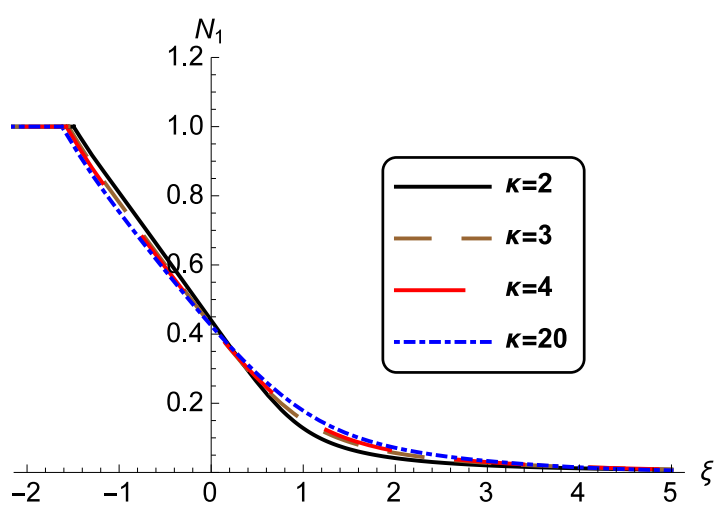

(c)

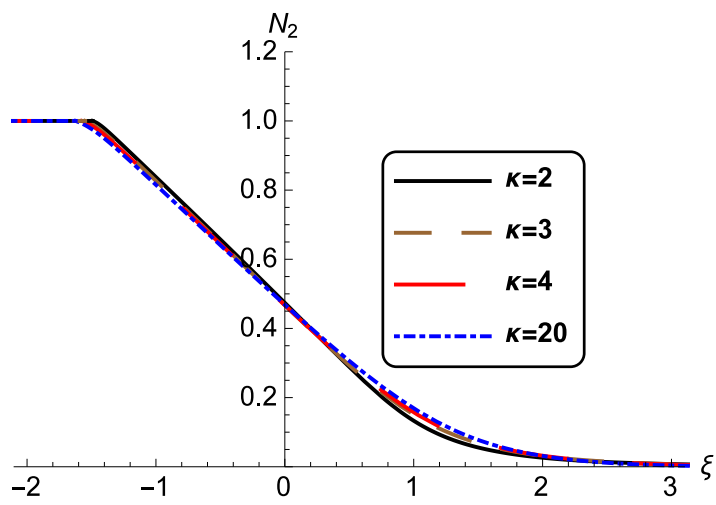

(e)

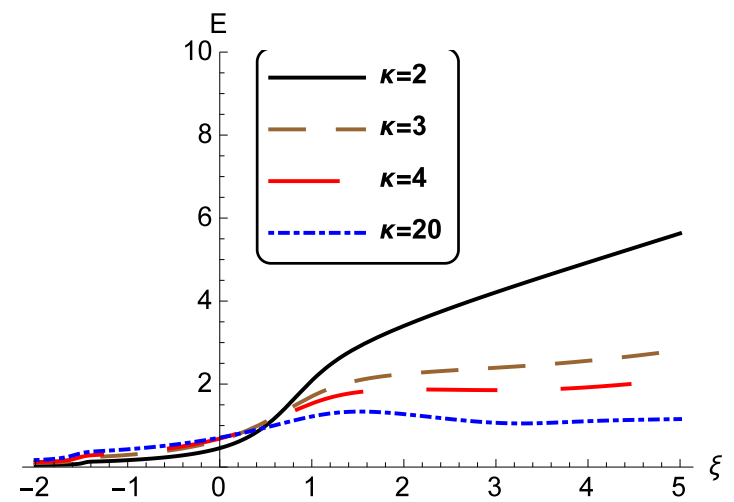

(b)

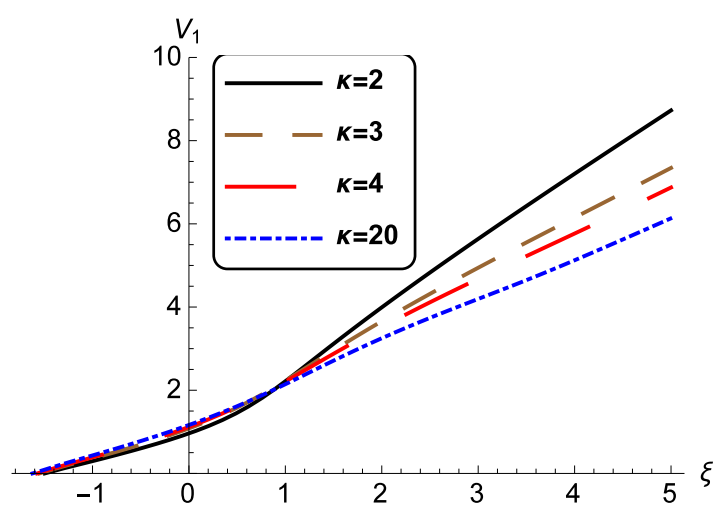

(d)

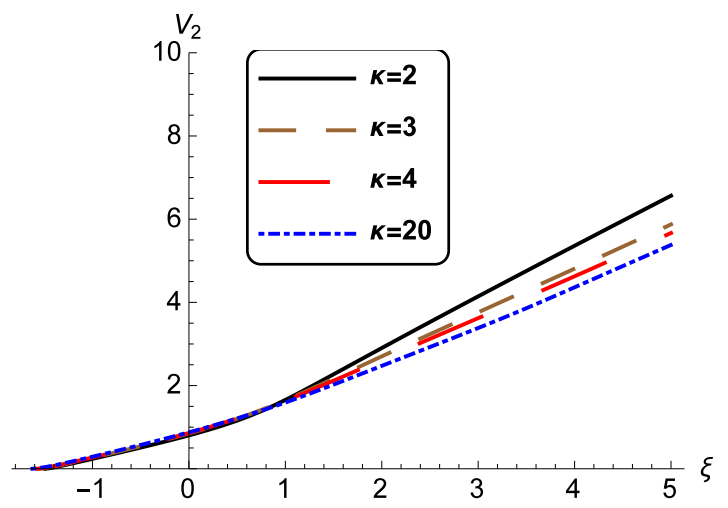

(f)

FIG. 8: (Color online) Electric field, electrostatic potential, density and velocity profiles of the two ion species with $\delta=1$, $\gamma=1 / 3$ and $\alpha_{1}=\alpha_{2}=1$ (just as in Fig. 7). Different values of $\kappa$ have been considered (see inset frame).

[23] J.E. Allen and J.G. Andrews, J. Plasma Physics, 4 (1), 187 (1970); J.E. Crow, P.L. Auer and J.E. Allen, J. Plasma Physics 14 (1), 65 (1975).

[24] P. Mora and R. Pellat, Phys. Fluids 22, 2300 (1979).

[25] P. Mora, Phys.Rev.Lett. 90, 185002 (2003).
[26] P. Mora, Phys. Plasmas 12, 112102 (2005)

[27] T. Grismayer, P. Mora, J. C. Adam, and A. Héron, Phys. Rev. E 77, 066407 (2008).

[28] P. Mora and T. Grismayer, Phys.Rev.Lett. 102, 145001 (2009). 


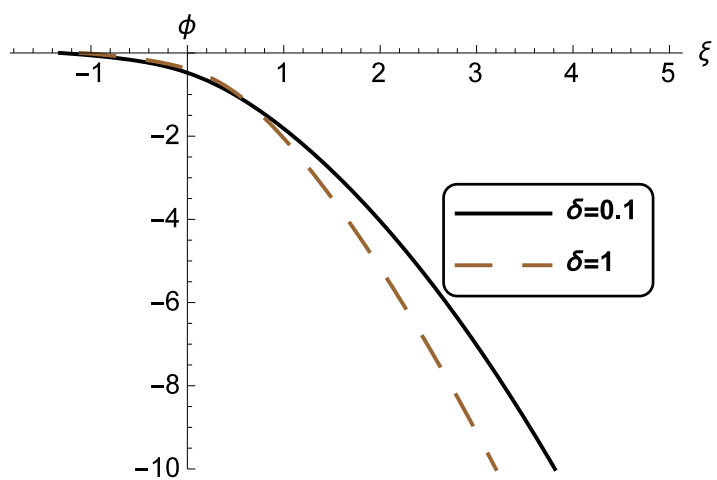

(a)

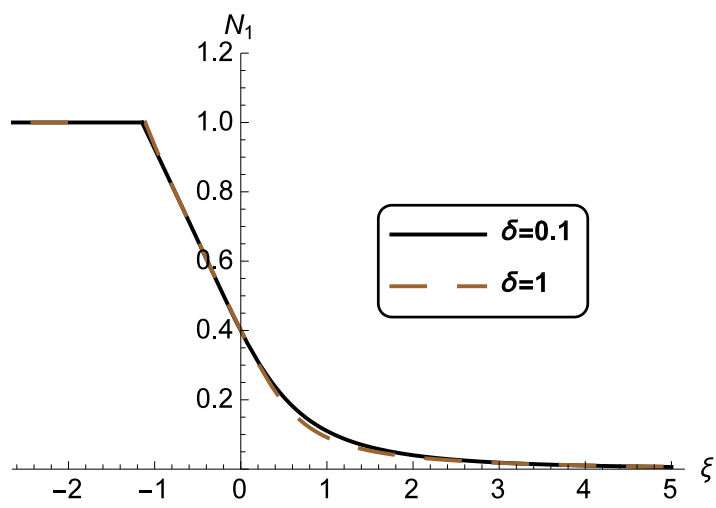

(c)

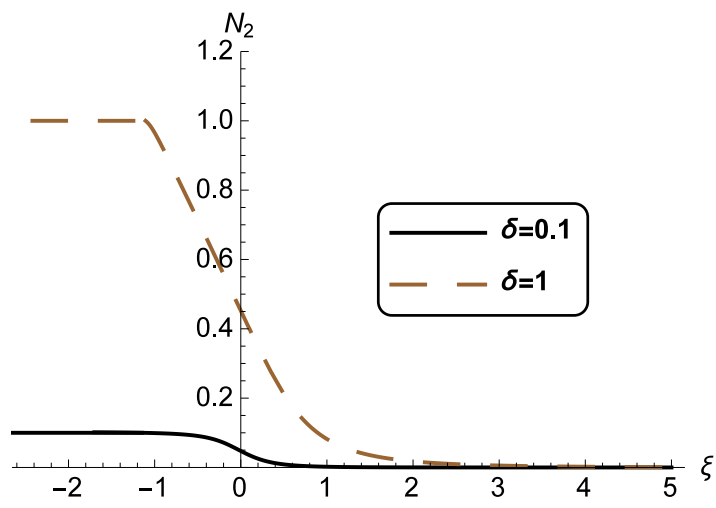

(e)

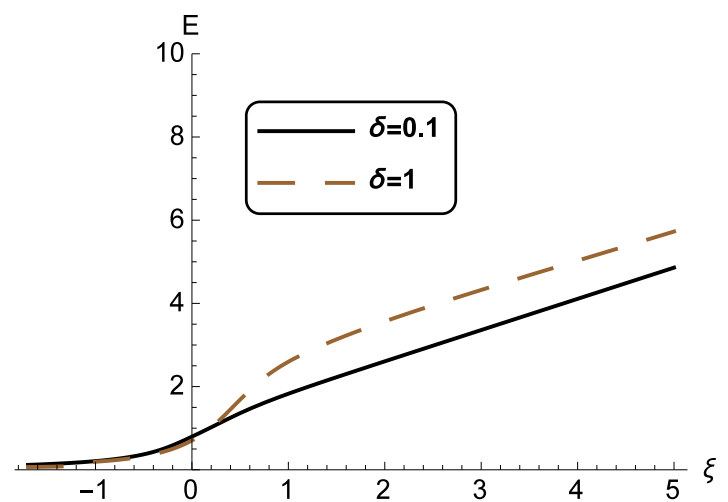

(b)

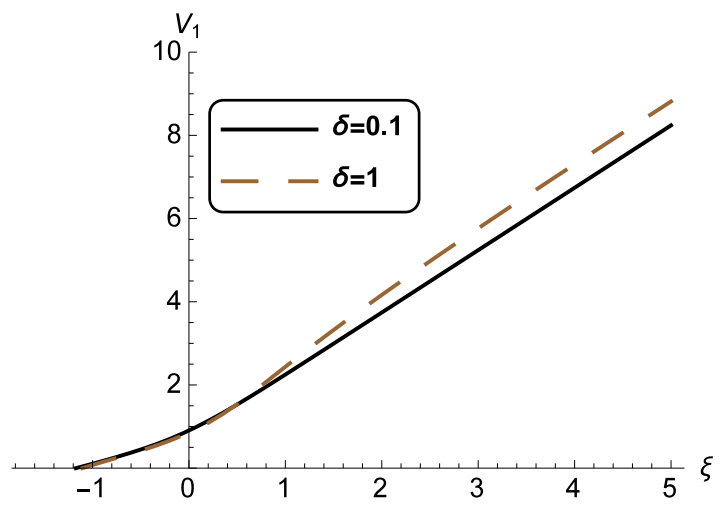

(d)

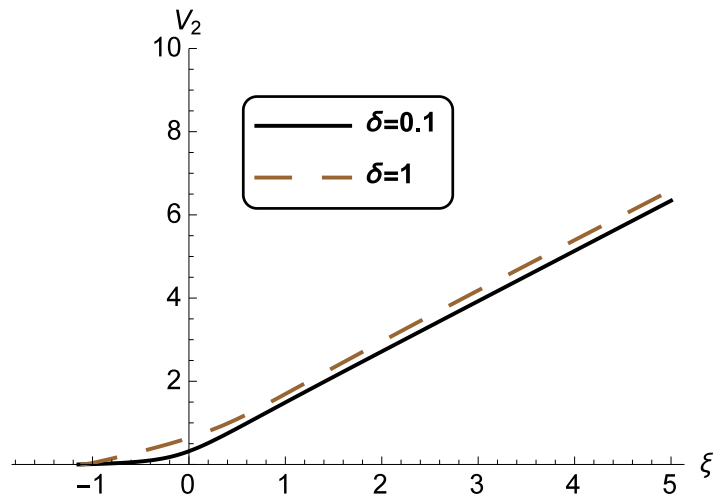

(f)

FIG. 9: (Color online) Electric field, electrostatic potential, density and velocity profiles of the two ion species with $\kappa=2$, $\gamma=1 / 3$ and $\alpha_{1}=\alpha_{2}=1$ i.e. $z_{1}=z_{2}$ and $m_{2}=3 m_{1}$. Different values of $\delta$ have been considered (see inset frame).

[29] P. Mora, Phys. Rev. E 91, 013107 (2015).

[30] S. Betti, F. Ceccherini, F. Cornolti and F. Pegoraro,Plasma Phys. Control. Fusion 47, 521 (2005).

[31] V. T. Tikhonchuk, A. A. Andreev, S. G. Bochkarev and V. Yu. Bychenkov, Plasma Phys. Control. Fusion 47, 869
$(2005)$.

[32] A. Diaw and P.Mora, Phys. Rev. E 84, 036402 (2011).

[33] A. Diaw and P.Mora, Phys. Rev. E 86, 026403 (2012).

[34] T. Kiefer, T. Schlegel, and M. C. Kaluza, Phys. Rev. E 87, 043110 (2013). 


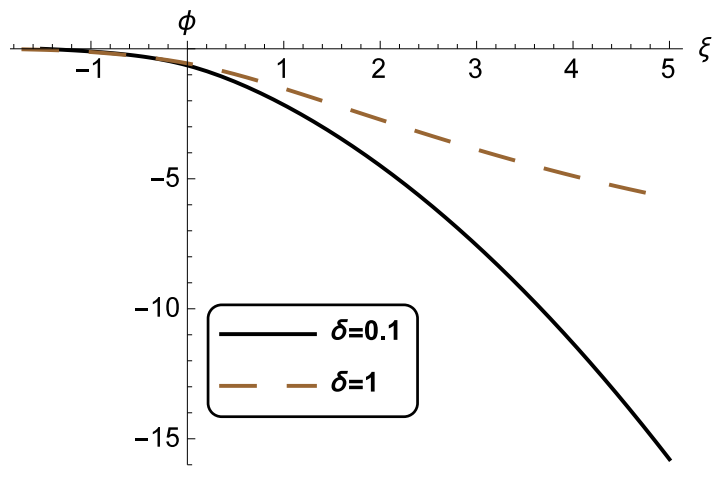

(a)

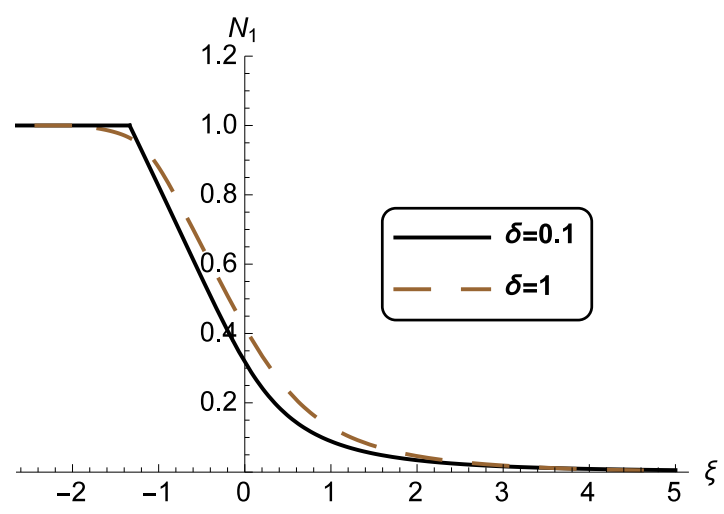

(c)

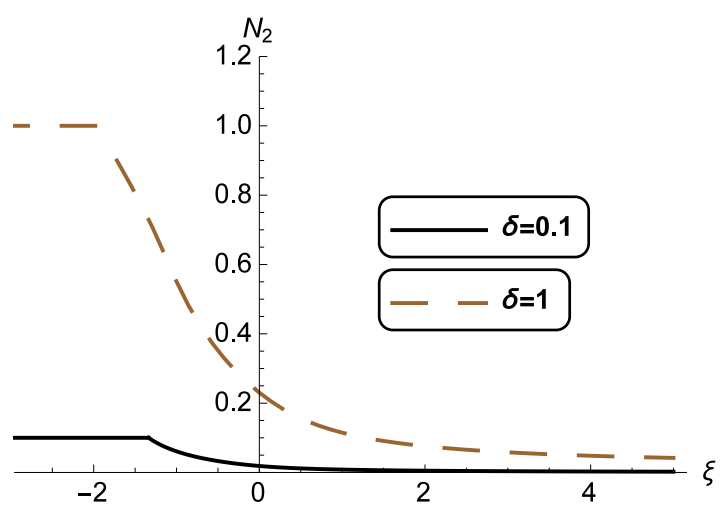

(e)

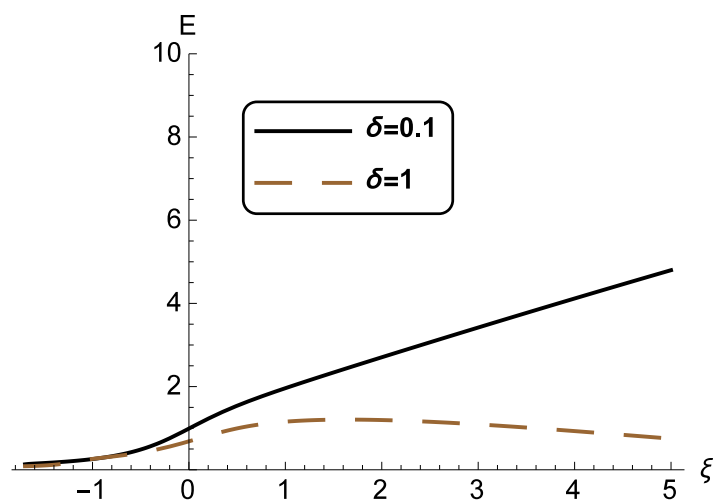

(b)

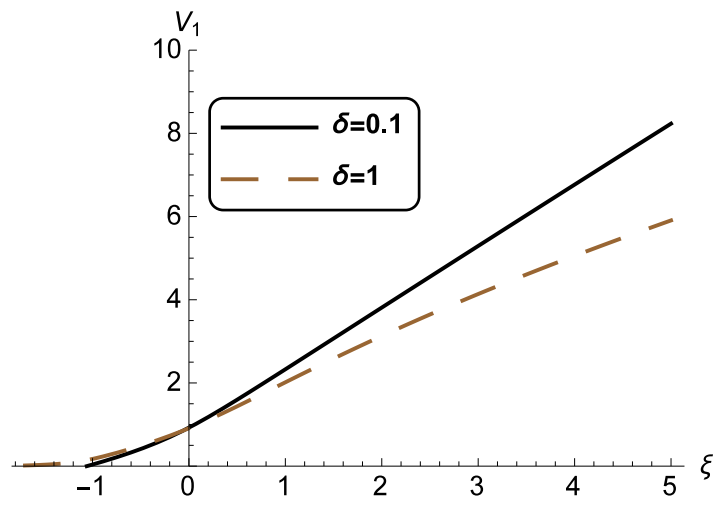

(d)

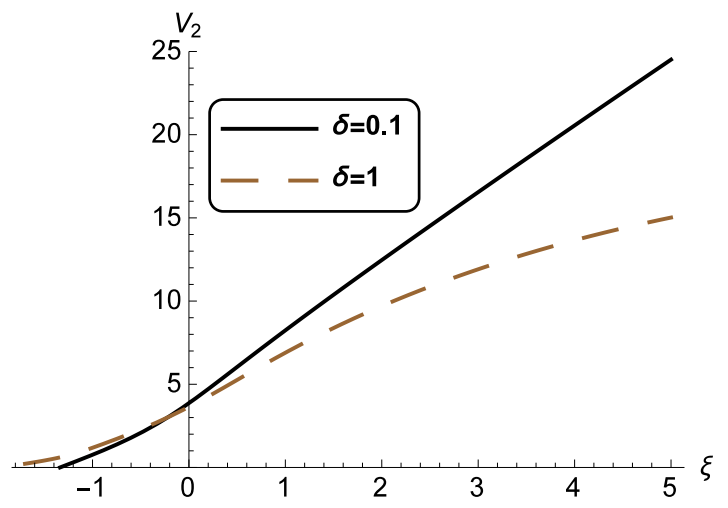

(f)

FIG. 10: (Color online) Electric field, electrostatic potential, density and velocity profiles of the two ion species with $\kappa=2$, $\gamma=16$ and $\alpha_{1}=\alpha_{2}=1$ i.e. $z_{1}=z_{2}, m_{1}=16 m_{2}$ and $n_{20}=n_{10}$.t Different values of $\delta$ have been considered (see inset frame).

[35] C. Thaury, P. Mora, A. Hron, J. C. Adam, and T. M. Antonsen Phys. Rev. E 82, 026408 (2010).

[36] C. Thaury, P. Mora, A. Hron, and J. C. Adam Phys. Rev. E 82, 016408 (2010).

[37] M. A. Hellberg, R. L. Mace, T. K. Baluku, I. Kourakis, and N. S. Saini, Phys. Plasmas 16, 094701 (2009).

[38] I. Kourakis, S. Sultana, and M. A. Hellberg, Plasma Phys. Controlled Fusion 54, 124001 (2012).

[39] V. Pierrard and M. Lazar, Solar Phys. 267, 153 (2010).

[40] G. Livadiotis and D. J. McComas, Space Science Re- 


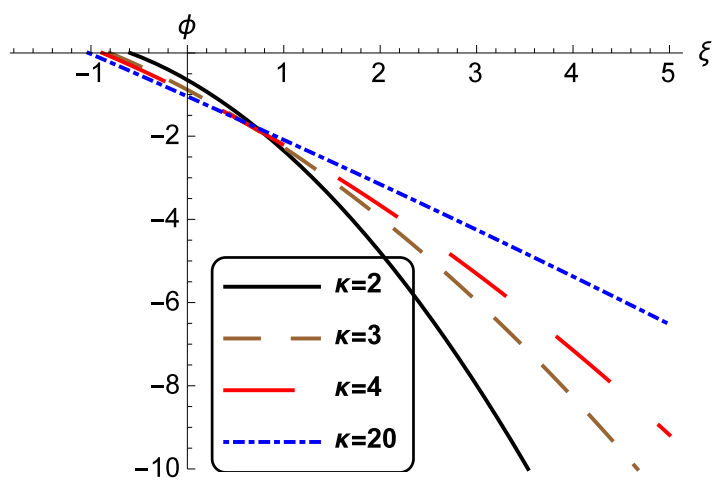

(a)

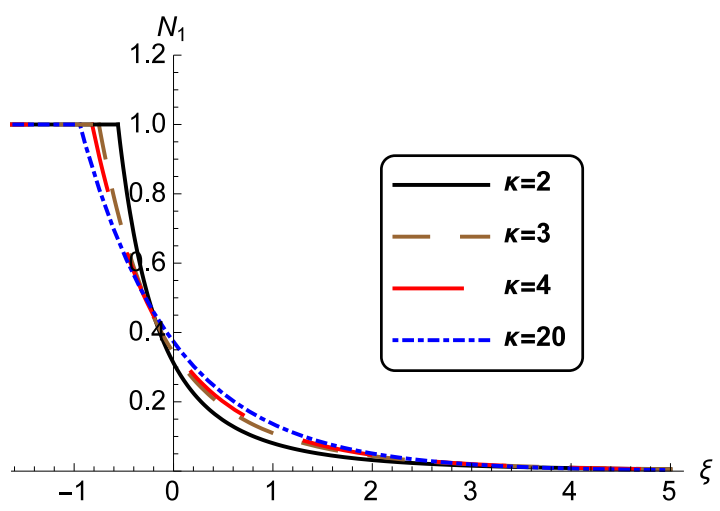

(c)

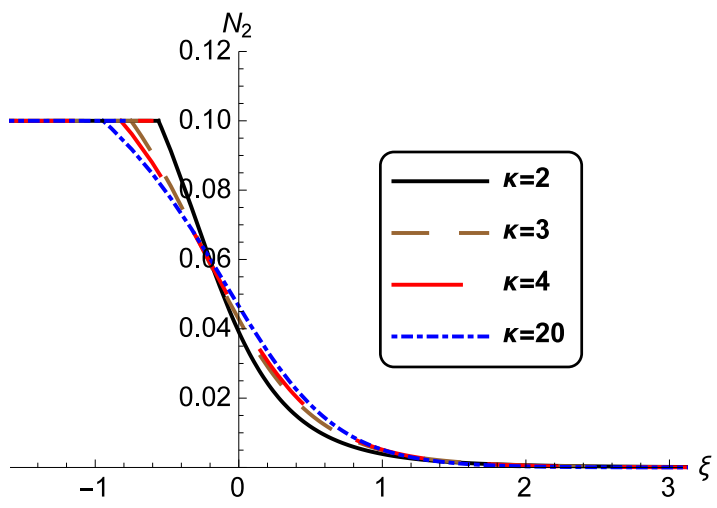

(e)

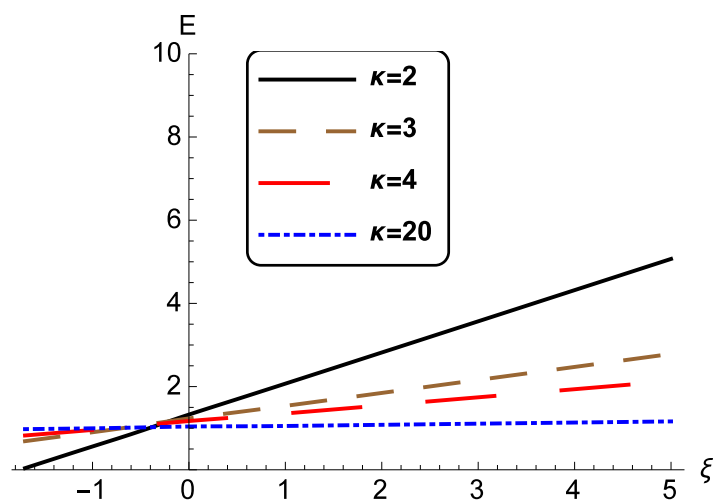

(b)

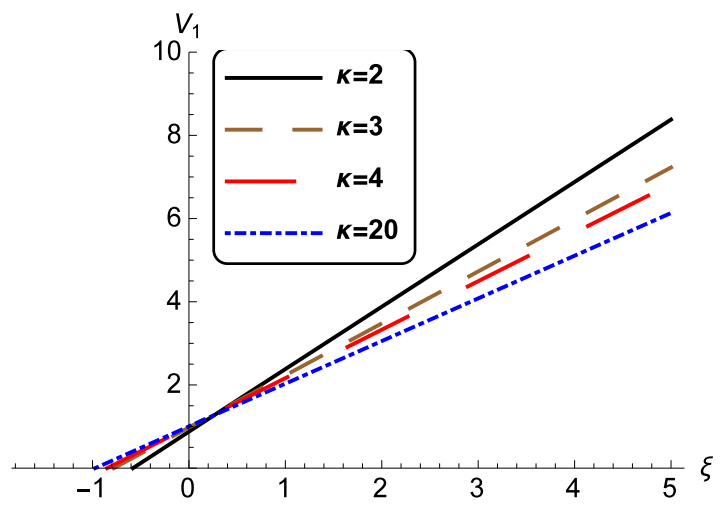

(d)

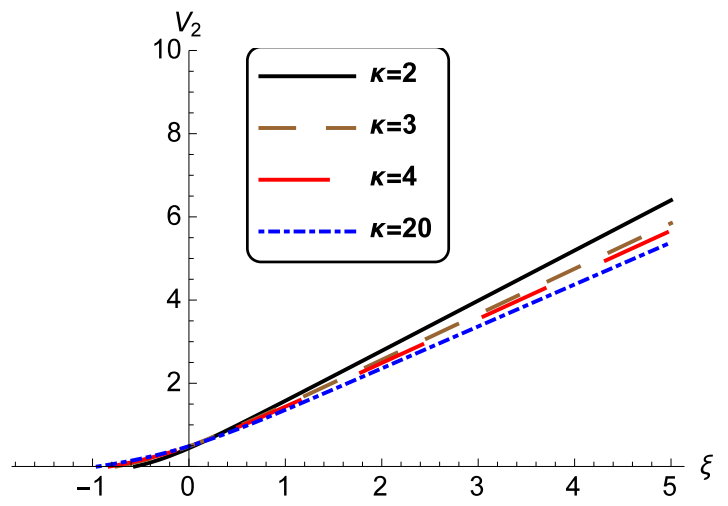

(f)

FIG. 11: (Color online) Electric field, electrostatic potential, density and velocity profiles of the two ion species with $\delta=0.1$, $\gamma=1 / 3$ and $\alpha_{1}=\alpha_{2}=0$, i.e. $z_{1}=z_{2}, m_{2}=3 m_{1}$ and $n_{20}=0.1 n_{10}$. Different values of $\kappa$ have been considered (see inset frame).

views, 175 (1), 183 (2013).

[41] G. Sarri, M.E. Dieckmann, C.R.D. Brown, C.A. Cecchetti, D.J.Hoarty, S.F.James, R.Jung, I.Kourakis, H.Schamel, O.Willi and M.Borghesi Phys. Plasmas, 17,
010701/1-4 (2010).

[42] D. Bennaceur-Doumaz, D. Bara, E. Benkhelifa and M. Djebli, J. Appl phys. 117, 043303 (2015).

[43] J. D. Menietti et al, J. Geophys. Res. 113 A05213 (2008). 
[44] P. Schippers et al, J. Geophys. Res. 113 A07208 (2008).

[45] R. Gaelzer et al, J. Geophys. Res. 115 A09109 (2010).

[46] G. Livadiotis et al, The Astrophysical Journal, 734, 1 (2011).

[47] M. Hapgood, C. Perry, J. Davies and M. Denton, Planet. Space Sci. 59618 (2011).

[48] P. H. Yoon, T. Rhee, and C. M. Ryu, Phys. Rev. Lett. 95, 215003 (2005)

[49] M.Borghesi, G.Sarri, C.A. Cecchetti, I. Kourakis, D. Hoarty, R.M.Stevenson, S.James, C.D.Brown, P.Hobbs, J.Lockyear, J.Morton, O.Willi, R. Jung and M.Dieckmann, Lasers and Particle Beams, 28 (2), 277 (2010).

[50] Marco Borghesi, Queen's University Belfast, private communication.

[51] S. P. Regan, N. B. Meezan, L. J. Suter, D. J. Strozzi, W. L. Kruer, D. Meeker, S. H. Glenzer, W. Seka, C. Stoeckl, V. Yu. Glebov, T. C. Sangster, D. D. Meyerhofer, R. L. McCrory, E. A. Williams, O. S. Jones, D. A. Callahan, M. D. Rosen, O. L. Landen, C. Sorce, and B. J. MacGowan, Phys. Plasmas 17, 020703 (2010).

[52] S.W.A. Irvine, S.C. Chapman and R.O. Dendy, Proc. 43rd EPS Conference on Plasma Physics (held in Leu- ven, Belgium), paper P5.096 in Europhysics Conference Abstracts Vol. 40A (ISBN 2-914771-99-1).

[53] M. Hegelich et al, Phys. Rev. Lett. 89, 085002 (2002).

[54] M. Allen et al, Phys. Rev. Lett. 93, 265004 (2004).

[55] B. Hegelich et al, Nature (London) 439, 441 (2006).

[56] Hamad Ahmed and Marco Borghesi (Queen's University Belfast), private communication).

[57] A.E. Turrell, M. Sherlock and S.J. Rose, Nature Comm. 6: 8905 (2015); DOI: 10.1038/ncomms9905.

[58] D. Anderson, J. Faulkner and H. Wilhelmsson, Physica Scripta 18, 141, (1978).

[59] A. P. L Robinson,A. R. Bell, and R. J. Kingham,Phys. Rev. Lett. 96, 035005 (2006).

[60] A.V. Gurevich, L.V. Pariiskaya, and L. P. Pitaevskii, Sov.Phys. JETP 36, 274 (1973).

[61] Y. V. Medvedev, Plasma Physics Reports 36, 507 (2010).

[62] J. Denavit, Phys. Fluids 22, 1384 (1979).

[63] N. Singh and R. W. Schunk, j. Geophys. Res 87, 9154 (1982); Phys.Fluids 26, 1123 (1983).

[64] The explicit assumption $N_{2}^{\prime} \ll N_{1}^{\prime}$ is a necessary condition for the analytical derivation of Eq. (38) [cf. (24, $25)$ in Ref. 58]; this (non-trivial) explicit assumption has been omitted in Ref. 58 . 